\section{Rate-Compatible Puncturing of Low-Density Parity-Check Codes}

\author{
Jeongseok Ha, Jaehong Kim, and
}

Steven W. McLaughlin, Senior Member, IEEE

\begin{abstract}
In this correspondence, we consider puncturing of low-density parity-check (LDPC) codes for additive white Gaussian noise (AWGN) channels. We show that good puncturing patterns exist and that the puncturing can be performed in a rate-compatible fashion. Furthermore, ratecompatible puncturing results in a small loss of performance with respect to threshold, namely, the punctured code is good (in terms of threshold) across a range of rates when compared with the optimal codes for each rate. This allows one to implement a single "mother" encoder and decoder that is good across a wide range of rates.
\end{abstract}

Index Terms-Capacity-approaching code, low-density parity-check (LDPC) code, puncturing.

\section{INTRODUCTION}

On time-varying channels, a common error control strategy is to adapt the rate according to available channel state information (CSI), which is called rate adaptability. An effective way to realize this coding strategy is to use a single code and puncture it in a rate-compatible fashion, a so-called rate-compatible punctured code (RCPC) [1]-[3]. In such an approach, the transmitter systematically punctures parity bits in a codeword, and the locations of the punctured symbols are known to the receiver/receivers. Since the decoder for the lowest rate (the base code) is compatible with ones for the other higher rates, RCPC needs no additional complexity for the rate adaptability. Moreover, RCPC permits one to transmit redundancies progressively in conjunction with automatic repeat request (ARQ) [1], [4].

In this correspondence, we apply this idea to low-density paritycheck (LDPC) codes, namely, one would like to have a single LDPC code which, when punctured in a rate-compatible way, remains good across a range of rates. We present a way to puncture LDPC codes which does not disturb the optimality of the base code, and where the resulting punctured codes maintain threshold optimality across a range of rates. We focus mainly on asymptotic thresholds of the punctured LDPC codes instead of practical issues such as code performance with a short block length, number of iterations to achieve a saturated performance, and finite precision effects due to quantization.

An LDPC code can be defined by an $m \times n$ sparse parity-check matrix $\boldsymbol{H}$, where $m$ is a number of parity-check equations, and $n$ is a block length. $\boldsymbol{H}$ can be graphically represented by a bipartite graph [5] depicted in Fig. 1. On the graph, each column (row) of the sparse paritycheck matrix is denoted as a variable (check) node and represents a received symbol (parity-check equation).

While a parity-check matrix and a bipartite graph specify an instance of an LDPC code, an ensemble of LDPC codes can be described with a degree distribution pair $(\lambda(x), \rho(x))[6]$.

$$
\lambda(x)=\sum_{i=2}^{d_{l}} \lambda_{i} x^{i-1}\left(\rho(x)=\sum_{i=2}^{d_{r}} \rho_{i} x^{i-1}\right)
$$

Manuscript received July 18, 2002; revised March 8, 2004. The material in this correspondence was presented in part at the IEEE International Symposium on Information Theory, Yokohama, Japan, June/July 2003.

The authors are with the School of Electrical and Computer Engineering, Georgia Institute of Technology, Atlanta, GA, USA (e-mail: jsha@ece.gatech. edu; onil@ece.gatech.edu; swm@ece.gatech.edu).

Communicated by R. Koetter, Associate Editor for Coding Theory.

Digital Object Identifier 10.1109/TIT.2004.836667

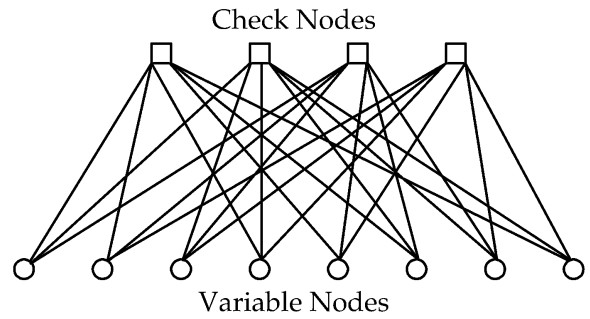

Fig. 1. A bipartite graph of a $(3,6)$ regular code.

is a polynomial whose coefficients are nonnegative real numbers, the sum of the coefficients is equal to $1, \lambda_{i}\left(\rho_{i}\right)$ is the fraction of edges belonging to variable (check) nodes with $i$ edges (or a degree of $i$ ), and $d_{l}\left(d_{r}\right)$ is the maximum variable (check) node degree. Thus, we can have many different parity-check matrices which comply with a degree distribution pair, and either a parity-check matrix or a bipartite graph is a realization of a degree distribution pair. The rate of LDPC codes specified by a degree distribution pair $(\lambda(x), \rho(x))$ is computed as (see [7])

$$
r(\lambda, \rho)=1-\frac{\int_{0}^{1} \rho(x) \mathrm{d} x}{\int_{0}^{1} \lambda(x) \mathrm{d} x}=1-\frac{\sum_{j=2}^{d_{r}} \rho_{j} / j}{\sum_{j=2}^{d_{l}} \lambda_{j} / j} .
$$

The degree distribution from an edge perspective can be converted to a node perspective with the following relations:

$$
\lambda_{j}^{\prime}=\frac{\lambda_{j} / j}{\sum_{i=2}^{d_{l}} \lambda_{i} / i} \Leftrightarrow \lambda_{j}=\frac{j \lambda_{j}^{\prime}}{\sum_{i=2}^{d_{l}} i \lambda_{i}^{\prime}}
$$

and

$$
\rho_{j}^{\prime}=\frac{\rho_{j} / j}{\sum_{i=2}^{d_{r}} \rho_{i} / i} \Leftrightarrow \rho_{j}=\frac{j \rho_{j}^{\prime}}{\sum_{i=2}^{d_{r}} i \rho_{i}^{\prime}}
$$

where $\lambda_{j}^{\prime}\left(\rho_{j}^{\prime}\right)$ is the fraction of variable (check) nodes having $j$ edges.

Richardson and Urbanke introduced the density evolution technique in [8] for evaluating thresholds of LDPC codes as a block length approaches infinity. The density evolution technique tells us a lower bound on the required signal-to-noise ratio (SNR) for error-free decoding averaged over all LDPC codes in an ensemble specified by a degree distribution pair.

For the puncturing problem, we shall set up the problem as follows. Variable nodes of a bipartite graph can be grouped in accordance with their edge degrees. Thus, all coded symbols with an edge degree $j$ belong to a group denoted as $G_{j}$ for $2 \leq j \leq d_{l}$. We shall randomly puncture a proportion $\pi_{j}^{(0)}$ of the symbols in $G_{j}$, where $\pi_{j}^{(0)}$ is determined with an optimization. We define $p^{(0)}$ to be the total puncturing fraction, namely $p^{(0)}=($ the number of punctured variable nodes $) /($ the number of variable nodes). We extend the distribution pair $(\lambda(x), \rho(x))$ to include a puncturing distribution, which is $\left(\lambda(x), \rho(x), \pi^{(0)}(x)\right)$, where

$$
\pi^{(0)}(x)=\pi_{2}^{(0)} x+\pi_{3}^{(0)} x^{2}+\cdots+\pi_{d_{l}}^{(0)} x^{d_{l}-1}
$$

and $0 \leq \pi_{j}^{(0)} \leq 1$. The puncturing fraction $p^{(0)}$ is expressed as

$$
p^{(0)}=\frac{\sum_{j=2}^{d_{l}} \pi_{j}^{(0)} n_{j}}{n}=\frac{\sum_{j=2}^{d_{l}} \pi_{j}^{(0)} \lambda_{j} / j}{\sum_{j=2}^{d_{l}} \lambda_{j} / j}=\sum_{j=2}^{d_{l}} \lambda_{j}^{\prime} \pi_{j}^{(0)}
$$




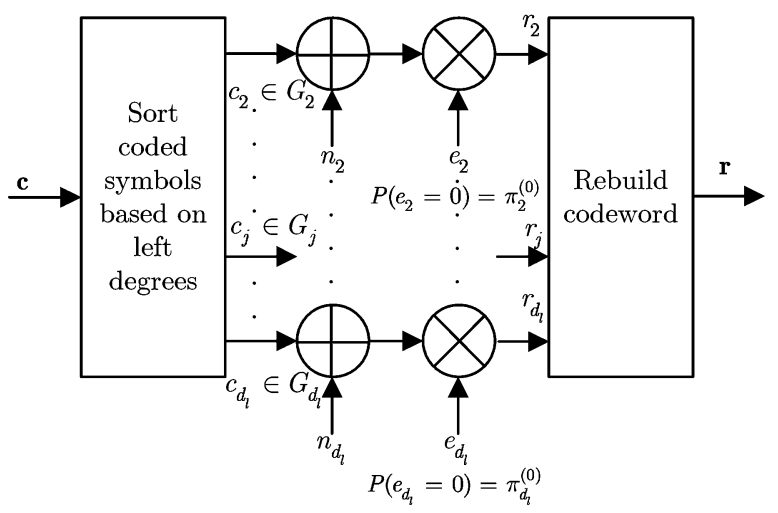

Fig. 2. Block diagram of the puncturing scheme, $\boldsymbol{c}$ is a codeword, $c_{j} \in\{-1,+1\}$ is a coded symbol in $G_{j}, \boldsymbol{r}$ is a received signal vector, $r_{j}$ is the received signal corresponding to $c_{j}, n_{j} \sim \mathcal{N}\left(0, \sigma_{n}^{2}\right), e_{j} \in\{0,1\}$, and $P\left(e_{j}=1\right)=1-P\left(e_{j}=0\right)$

where $n_{j}=\left|G_{j}\right|$ and $n=\sum_{j=2}^{d_{l}}\left|G_{j}\right|$. The rate of punctured LDPC codes specified by a three-tuple distribution $\left(\lambda(x), \rho(x), \pi^{(0)}(x)\right)$ is $r\left(\lambda, \rho, \pi^{(0)}\right)=r(\lambda, \rho) /\left(1-p^{(0)}\right)$. An ensemble of LDPC codes over additive white Gaussian noise (AWGN) channels and the mixed channel in [9] are special cases expressed by three-tuple distributions

$$
(\lambda(x), \rho(x), O(x)) \quad \text { and } \quad\left(\lambda(x), \rho(x), e^{(0)} I(x)\right)
$$

respectively, where

$$
\begin{aligned}
& O(x)=\sum_{j=2}^{d_{l}} 0 \cdot x^{j-1} \\
& I(x)=\sum_{j=2}^{d_{l}} x^{j-1}
\end{aligned}
$$

and $e^{(0)}$ is a uniform puncturing/erasure probability.

The proposed puncturing scheme is depicted in Fig. 2, and the probability density function of the received symbol $r_{j}$ corresponding to a coded symbol $c_{j}$ is shown in Fig. 3, where a proportion $\pi_{j}^{(0)}$ of the symbols in $G_{j}$ is punctured. Our goal is to design puncturing proportions $\pi_{j}^{(0)}$ 's for all $j$, which optimizes (minimizes) the SNR threshold for a given puncturing fraction $p^{(0)}$. An equivalent optimization is to fix the SNR threshold and to maximize the puncturing fraction $p^{(0)}$.

The basic idea is as follows: assume that a base (unpunctured) code has been designed for rate $R$. Since the punctured version of that code has rate $R^{\prime}>R$, its SNR threshold is higher. So rather than fix the puncturing fraction, we fix the target SNR threshold of the punctured code and optimize (maximize) the puncturing fraction $p^{(0)}$ and puncturing proportions $\pi_{j}^{(0)}$ 's for all $j$ for that threshold. As we shall see shortly, the result is a sequence of punctured codes that have thresholds close to those of a set of codes optimally designed for each rate.

For the variable nodes in $G_{j}$, the probability density function of their log-likelihood ratio (LLR) message can be expressed as

$$
\begin{aligned}
f\left(v_{j}\right) & =\frac{1-\pi_{j}^{(0)}}{\sqrt{4 \pi m_{u_{0}}}} e^{-\frac{\left(v_{j} \pm m_{u_{0}}\right)^{2}}{4 m_{u_{0}}}}+\pi_{j}^{(0)} \Delta_{0}\left(v_{j}\right) \\
& =g^{(0)}\left(v_{j}\right)+\pi_{j}^{(0)} \Delta_{0}\left(v_{j}\right)
\end{aligned}
$$

where $v_{j}=\log _{e}\left[p\left(r_{j} \mid c_{j}=+1\right) / p\left(r_{j} \mid c_{j}=-1\right)\right], m_{u_{0}}=E\left[v_{j} \mid v_{j} \neq\right.$ $0]=E\left[2 r_{j} / \sigma_{n}^{2} \mid r_{j} \neq 0\right], \operatorname{Var}\left(v_{j} \mid v_{j} \neq 0\right)=2 m_{u_{0}}, \pi_{j}^{(0)}$ is the puncturing proportion of symbols in $G_{j}$, and $\Delta_{x}\left(v_{j}\right)=\delta\left(v_{j}-x\right)$ is a shifted delta function.

This correspondence is organized into four sections. In Section I, we have introduced the channel model and defined terminology for the subsequent sections. In Section II, we briefly summarize Chung's

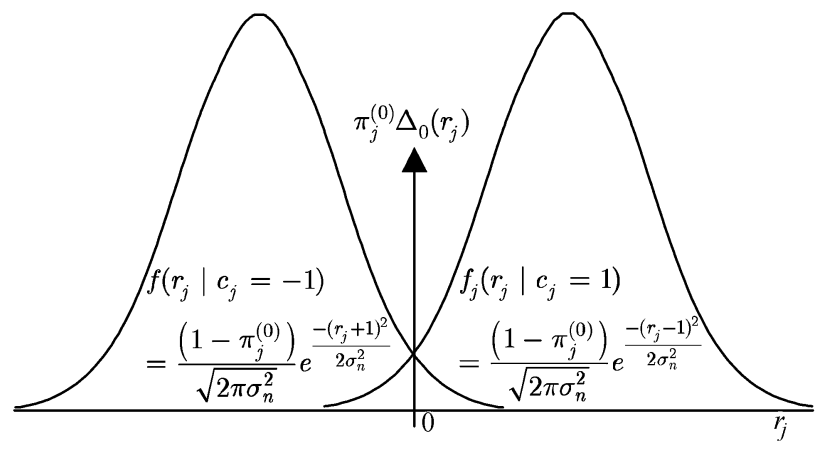

Fig. 3. Probability density function of the received symbol corresponding to $c_{j}, c_{j} \in\{-1,+1\}$ is a coded symbol in $G_{j}, \pi_{j}^{(0)}$ is a puncturing proportion, $r_{j}$ is a received symbol, and $\sigma_{n}$ is the standard deviation of the white Gaussian noise.

work in [10] for the reader's convenience. In Section III, we analyze the thresholds of punctured LDPC codes with Gaussian approximation (GA) in [10]. The analytic results are useful to understand the convergence of punctured LDPC codes and can be further simplified with a proper assumption. The simplified recursive equation, called a steadystate equation, tells us how punctured LDPC codes perform and how to design the puncturing distributions. Thus, the analysis gives us not only a prediction method of the thresholds of punctured LDPC codes but also a design rule for good puncturing distributions. In Section IV, we design puncturing distributions for two LDPC codes from [7] and [11]. We also implement these LDPC codes with a block length of 131072 and puncture them according to the designed puncturing distributions. Through simulations, we confirm consistency between the asymptotic and implemented performance. Finally, in Section V, we summarize our work.

\section{GAUSSIAN APPROXIMATION}

Our work is based on the results of GA in [10]. In this section, we briefly summarize GA. This summary is the minimum needed to understand our work and describes GA for irregular LDPC codes, which includes regular LDPC codes as a special case.

The LLR message (message for short) of a check node at the $k$ th iteration $\left(u^{(k)}\right.$ and $k \in \mathbb{N}$ ) during message-passing decoding is approximated as a Gaussian random variable that is completely specified by its mean $\left(m_{u}^{(k)}\right)$ and variance $\left(\operatorname{Var}\left(u^{(k)}\right)\right)$. The symmetry condition, $f(x)=e^{x} f(-x)$, which is preserved during iterations, imposes the following relation between the mean and variance: $\operatorname{Var}\left(u^{(k)}\right)=$ $2 m_{u}^{(k)}$. Thus, by tracing the changes of the mean (called updated mean) during iterations, we can watch the evolution of the probability density function (density for short) of the check node message, $f^{(k)}(u)=$ $\mathcal{N}\left(m_{u}^{(k)}, 2 m_{u}^{(k)}\right)$, where $\mathcal{N}\left(\mu, \sigma^{2}\right)$ is the normal probability density function with a mean $\mu$ and a variance $\sigma^{2}$.

The density of a variable node message at the $k$ th iteration $\left(v^{(k)}\right)$ is approximated as a Gaussian mixture for irregular LDPC codes and a Gaussian for regular LDPC codes

$$
f^{(k)}(v)=\sum_{i=2}^{d_{l}} \lambda_{i} \mathcal{N}\left(m_{v, i}^{(k)}, 2 m_{v, i}^{(k)}\right)
$$

where $\lambda_{i}$ is the fraction of edges belonging to degree $i$ variable nodes, $m_{v, i}^{(k)}=m_{u_{0}}+(i-1) m_{u}^{(k-1)}, m_{u_{0}}$ is the mean of an LLR message out of channel, and $m_{u}^{(0)}=0$.

Between a check node of degree $d_{c}$ and the neighboring variable nodes, there is the well-known tanh rule [12]

$$
\tanh \frac{u^{(k)}}{2}=\prod_{j=1}^{d_{c}-1} \tanh \frac{v_{j}^{(k)}}{2}
$$


where $v_{j}^{(k)}, j=1, \ldots, d_{c}-1$, are the incoming messages from $d_{c}-1$ neighboring variable nodes and $u^{(k)}$ is the message sent to the remaining variable node. Since the variable node messages are assumed to be independent and identically distributed, we can omit the index $j$ in (3) and simplify the product as

$$
E\left[\tanh \frac{u^{(k)}}{2}\right]=E\left[\tanh \frac{v^{(k)}}{2}\right]^{d_{c}-1} .
$$

The expectation $E\left[\tanh \frac{u}{2}\right]$ can be expressed as

$$
E\left[\tanh \frac{u}{2}\right]=\frac{1}{\sqrt{4 \pi m_{u}}} \int_{\mathbb{R}} \tanh \frac{u}{2} e^{\frac{-\left(u-m_{u}\right)^{2}}{4 m_{u}}} d u
$$

where $u$ is a Gaussian random variable with $m_{u}=E[u]$ and $\operatorname{Var}(u)=$ $2 m_{u}$. Thus, both sides of (4) become integral forms that are simplified by the following definition.

Definition 1 ([10, Definition 1]):

$$
\phi(x)= \begin{cases}1-\frac{1}{\sqrt{4 \pi x}} \int_{\mathbb{R}} \tanh \frac{u}{2} e^{\frac{-(u-x)^{2}}{4 x}} d u, & \text { if } x>0 \\ 1, & \text { if } x=0 .\end{cases}
$$

$\phi(x)$ is continuous and monotonically decreasing on $[0, \infty)$, with $\phi(0)=1$ and $\phi(\infty)=0$.

By applying (5) and $\phi(x)$ to both sides of (4), we can calculate the updated mean of a degree $j$ check node message as

$$
m_{u, j}^{(k)}=\phi^{-1}\left(1-\left[1-\sum_{i=2}^{d_{l}} \lambda_{i} \phi\left(m_{v, i}^{(k)}\right)\right]^{(j-1)}\right) .
$$

We have a recursive equation for the updated mean

$$
\begin{aligned}
m_{u}^{(k)}= & \sum_{j=2}^{d_{r}} \rho_{j} m_{u, j}^{(k)} \\
= & \sum_{j=2}^{d_{r}} \rho_{j} \phi^{-1} \\
& \times\left(1-\left[1-\sum_{i=2}^{d_{l}} \lambda_{i} \phi\left(m_{u_{0}}+(i-1) m_{u}^{(k-1)}\right)\right]^{(j-1)}\right) .
\end{aligned}
$$

For error-free decoding, the updated mean converges to $\infty$ as $k \rightarrow \infty$, which implies

$$
\sum_{i=2}^{d_{l}} \lambda_{i} \phi\left(m_{u_{0}}+(i-1) m_{u}^{(k-1)}\right) \rightarrow 0, \quad \text { as } k \rightarrow \infty
$$

since $\phi^{-1}(x) \rightarrow \infty$ as $x \rightarrow 0$.

For $0 \leq s<\infty$ and $0<r \leq 1, h_{i}(s, r)$ and $h(s, r)$ are defined in $[10]$ as

$$
\begin{aligned}
h_{i}(s, r) & =\phi\left(s+(i-1) \sum_{j=2}^{d_{r}} \rho_{j} \phi^{-1}\left(1-(1-r)^{j-1}\right)\right) \\
h(s, r) & =\sum_{i=2}^{d_{l}} \lambda_{i} h_{i}(s, r)
\end{aligned}
$$

and (6) is equivalently expressed as

$$
r_{k}=h\left(s, r_{k-1}\right)
$$

where $s=m_{u_{0}}$ and $r_{0}=\phi\left(m_{u_{0}}\right)$.

In the recursion, the degree distribution pair $(\lambda(x), \rho(x))$ and $s$ determine the convergence to a fixed point (not necessarily zero). As shown in (6) and (7), $m_{u}^{(k)} \rightarrow \infty$ iff $r_{k} \rightarrow 0$. Thus, the convergence condition $r_{k}(s) \rightarrow 0$ is satisfied iff

$$
h(s, r)<r \quad \forall r \in(0, \phi(s)) .
$$

Definition 2 ([10, Definition 2]): The threshold $s^{*}$ is the infimum of all $s$ in $\mathbb{R}^{+}$such that $r_{k}(s)\left(m_{u}^{(k)}(s)\right)$ converges to $0(\infty)$ as $k \rightarrow \infty$.

In [10], $\phi(x)$ is approximated as

$$
\phi(x) \approx \begin{cases}e^{\alpha x^{\gamma}+\beta}, & \text { for } 0 \leq x<10 \\ \sqrt{\frac{\pi}{x}} e^{-\frac{x}{4}}\left(1+\frac{1}{14 x}-\frac{3}{2 x}\right), & \text { for } 10 \leq x\end{cases}
$$

where $\alpha=-0.4527, \beta=0.0218$, and $\gamma=0.8600$. The approximation of $\phi(x)$ is accurate enough at all the values of $x$ except around $x=0$, where $\phi(0)=1$ but $e^{\beta}>1$. In the process of designing LDPC codes over the AWGN channel, $\phi(x)$ in $h_{j}(m, r)$ is computed only at $x \geq m>0$. Thus, the approximation is good enough. However, we will show that for the analysis of punctured LDPC codes we have to compute $\phi(x)$ around $x=0$. Therefore, we modify the approximation as

$$
\phi(x) \approx \begin{cases}e^{-a x^{2}-b x}, & \text { for } 0 \leq x<c \\ e^{\alpha x^{\gamma}+\beta}, & \text { for } c \leq x<10 \\ \sqrt{\frac{\pi}{x}} e^{-\frac{x}{4}}\left(1+\frac{1}{14 x}-\frac{3}{2 x}\right), & \text { for } 10 \leq x\end{cases}
$$

where $a=-0.0564, b=0.48560$, and $c=0.867861$. The parameters, $a, b$, and $c$ are acquired by curve-fitting.

\section{Puncturing Analysis With GA}

The message flows between check nodes and variable nodes are depicted in Fig. 4(a) and (b) where the square and the circle symbols represent check and variable nodes, respectively. Assuming the decoder knows the positions of the punctured symbols, it will set the LLRs of the punctured symbols to zero at the initialization. The function $g^{(k)}(v)\left(\left(1-\varepsilon^{(k)}\right) h^{(k)}(u)\right)$ is the continuous part of the density of a variable (check) node message. The term $e^{(k)}\left(\varepsilon^{(k)}\right)$ is the probability for a variable (check) node LLR message to be equal to zero, $d_{v}\left(d_{c}\right)$ is the number of neighbors of a variable (check) node, and $v_{j}^{(k)}\left(u_{j}^{(k)}\right)$ is the message of a variable (check) node emitting through the $j$ th edge (we will drop the edge index $j$ without loss of generality hereafter). In the sum-product decoding algorithm, if at least one of the variable nodes connected to a check node is punctured, the check node has zero LLR as its message. Thus, $\varepsilon^{(k)}$ is expressed as

$$
\begin{aligned}
\varepsilon^{(k)} & =\sum_{s=2}^{d_{r}} P\left(d_{c}=s\right) \varepsilon_{d_{c}=s}^{(k)} \\
& =\sum_{s=2}^{d_{r}} \rho_{s}\left(1-\left(1-e^{(k)}\right)^{s-1}\right)=1-\rho\left(1-e^{(k)}\right)
\end{aligned}
$$




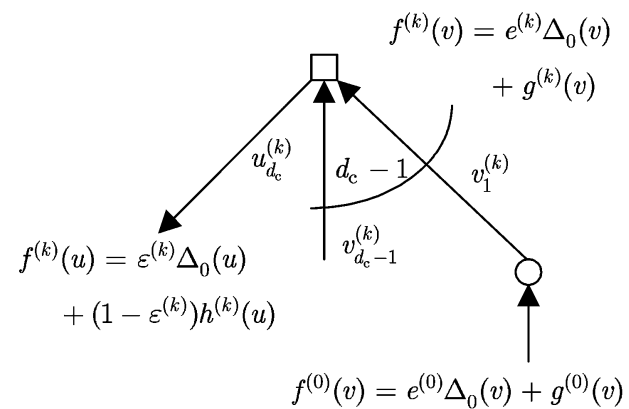

(a)

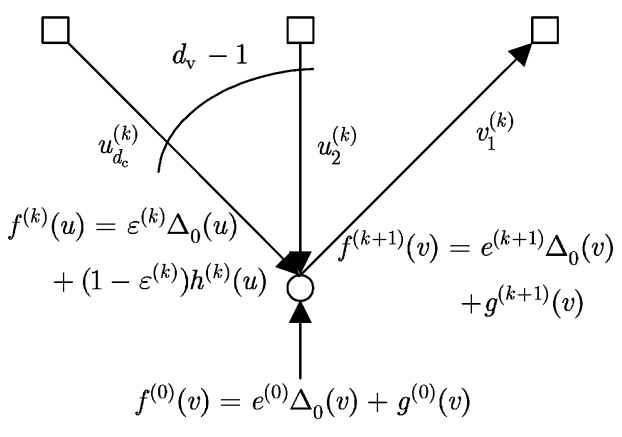

(b)

Fig. 4. Message flows between a check node and a variable node: (a) LLR message of a check node at the $\boldsymbol{k}$ th iteration. (b) LLR message of a variable node at the $(k+1)$ th iteration.

where

$$
\begin{aligned}
\varepsilon_{d_{c}=s}^{(k)} & =P\left(u^{(k)}=0 \mid d_{c}=s\right) \\
& =1-\prod_{i=1}^{s-1} P\left(v^{(k)} \neq 0\right)=1-\left(1-e^{(k)}\right)^{s-1}
\end{aligned}
$$

and $\varepsilon_{d_{c}=s}^{(k)}=P\left(u^{(k)}=0 \mid d_{c}=s\right)$ is the probability that a check node having $s$ edges sends a zero LLR message to variable nodes connected to the check node.

If none of the variable nodes connected to a check node are punctured, the probabilistic characteristics of the check message can be approximated as Gaussian that is denoted as $h^{(k)}(u)$ in Fig. 4(a) and (b). The Gaussian density of an LLR message is determined by one variable, the updated mean value $m_{u}^{(k)}$ at the $k$ th iteration. It is determined from the following relation:

$$
\begin{aligned}
m_{u}^{(k)} & =\sum_{s=2}^{d_{c}} P\left(d_{c}=s\right) m_{u \mid d_{c}=s}^{(k)} \\
& =\sum_{s=2}^{d_{c}} \rho_{s} m_{u \mid d_{c}=s}^{(k)}
\end{aligned}
$$

where

$$
\begin{aligned}
m_{u \mid d_{c}=s}^{(k)} & =\phi^{-1}\left(1-E\left[\tanh \left[u^{(k)} / 2\right] \mid u^{(k)} \neq 0, d_{c}=s\right]\right) \\
& =\phi^{-1}\left(1-E\left[\tanh \left[v^{(k)} / 2\right] \mid v^{(k)} \neq 0\right]^{s-1}\right)
\end{aligned}
$$

and $m_{u \mid d_{c}=s}^{(k)}$ is the conditional updated mean of a check node message given the number of its edges is $s$.

We can express the updated mean value as

$$
\begin{aligned}
m_{u}^{(k)}=\sum_{s=2}^{d_{r}} \rho_{s} \phi^{-1}(1 & -\frac{1}{\left(1-e^{(k)}\right)^{s-1}} \\
& \left.\times\left\{\left\langle\tanh \left[v^{(k)} / 2\right], g^{(k)}(v)\right\rangle\right\}^{s-1}\right)
\end{aligned}
$$

and

$$
\begin{aligned}
& E\left[\tanh \left[v^{(k)} / 2\right] \mid v^{(k)} \neq 0\right]^{s-1} \\
& =\frac{1}{\left(1-e^{(k)}\right)^{s-1}}\left\{\left\langle\tanh \left[v^{(k)} / 2\right], g^{(k)}(v)\right\rangle\right\}^{s-1}
\end{aligned}
$$

where $\langle f(x), g(x)\rangle=\int_{\mathbb{R}} f(x) g(x) \mathrm{d} x$.

To make (9) a recursive equation of $m_{u}^{(k)}$, we need an expression for the density of a variable node message in terms of $m_{u}^{(k-1)}$. In the log-probability domain, the variable node message is determined by the linear sum of $d_{v}-1$ incident check node messages as shown in Fig. 4. Thus, the probability that the message of a variable node is zero can be computed as

$$
\begin{aligned}
e^{(k)} & =P\left(v^{(k)}=0\right) \\
& =\sum_{j=2}^{d_{l}} P\left(d_{v}=j\right) e_{j}^{(k)} \\
& =\sum_{j=2}^{d_{l}} \lambda_{j} \pi_{j}^{(0)}\left(\varepsilon^{(k-1)}\right)^{j-1} \\
& =\lambda^{\pi}\left(\varepsilon^{(k-1)}\right)=\lambda^{\pi}\left(1-\rho\left(1-e^{(k-1)}\right)\right)
\end{aligned}
$$

where $e_{j}^{(k)}$ is the conditional probability that all incident check node messages are zero given the variable node has $j$ edges, $e_{j}^{(0)}=\pi_{j}^{(0)}$, $\varepsilon^{(-1)}=1, \lambda^{\pi}(x)=\sum_{j=2}^{d_{l}} \lambda_{j}^{\pi} x^{j-1}$, and $\lambda_{j}^{\pi}=\lambda_{j} \pi_{j}^{(0)}$.

The residual puncturing proportion $\pi_{j}^{(k)}$ and fraction $p^{(k)}$ at the $k$ th iteration can be computed as

$$
\begin{aligned}
& \pi_{j}^{(k)}=\pi_{j}^{(0)}\left(\varepsilon^{(k-1)}\right)^{j}, \text { and } \\
& p^{(k)}=\sum_{j=2}^{d_{l}} \lambda_{j}^{\prime} \pi_{j}^{(k)} .
\end{aligned}
$$

Thus, the puncturing fraction $p^{(0)}$ in $(2)$ is $p^{(k)}$ at $k=0$ with $\varepsilon^{(-1)}=1$.

The density of a variable node message can be expressed as a sum of three terms as shown in (12) at the bottom of the page. The first term gives the probability in (10), the second term describes the incident check node messages given the variable node is punctured, and the last

$$
\begin{aligned}
f^{(k)}(v)= & e^{(k)} \Delta_{0}(v)+g^{(k)}(v) \\
= & e^{(k)} \Delta_{0}(v)+\left\{\sum_{j=2}^{d_{l}} \lambda_{j}^{\pi} \sum_{i=1}^{j-1}(j-1) \chi_{i}^{(k)} \mathcal{N}\left(i m_{u}^{(k-1)}, 2 i m_{u}^{(k-1)}\right)\right\} \\
& +\left\{\sum_{j=2}^{d_{l}} \lambda_{j}^{(1-\pi)} \sum_{i=0}^{j-1}(j-1) \chi_{i}^{(k)} \mathcal{N}\left(i m_{u}^{(k-1)}+m_{u_{0}}, 2 i m_{u}^{(k)}+2 m_{u_{0}}\right)\right\}
\end{aligned}
$$


term is the combination of the check node messages with the unpunctured received message. The continuous part used in (9) comprises the last two terms, where

$$
\begin{aligned}
\lambda_{j}^{(1-\pi)} & =\left(1-\pi_{j}^{(0)}\right) \lambda_{j} \\
\mathcal{N}(m, 2 m) & =\frac{1}{\sqrt{4 \pi m}} e^{-\frac{(v-m)^{2}}{4 m}} \\
{ }_{n} \chi_{m}^{k} & ={ }_{n} \mathrm{C}_{m}\left(\varepsilon^{(k-1)}\right)^{n-m}\left(1-\varepsilon^{(k-1)}\right)^{m}
\end{aligned}
$$

and ${ }_{n} \mathrm{C}_{m}$ is the binomial coefficient. The continuous part of a variable node message density derived in (12) expands (9) to (13) at the bottom of the page, where

$$
\begin{aligned}
\langle\tanh (v / 2), \mathcal{N}(x, 2 x)\rangle & =\int_{\mathbb{R}} \tanh (v / 2) \frac{1}{\sqrt{4 \pi x}} e^{-\frac{(v-x)^{2}}{4 x}} d v \\
& =1-\phi(x) .
\end{aligned}
$$

The updated mean becomes a recursive equation in (13).

Since we model the message densities as Gaussian, the bit error probability can be computed as a weighted sum of $Q$ functions as follows:

$$
\begin{aligned}
P_{e}^{(k)}= & \sum_{j=2}^{d_{l}} \lambda_{j}^{\prime} \pi_{j}^{(0)} \sum_{i=0}^{j}{ }_{j} \chi_{i}^{(k)} Q\left(\sqrt{\frac{i m_{u}^{(k)}}{2}}\right) \\
& +\sum_{j=2}^{d_{l}} \lambda_{j}^{\prime}\left(1-\pi_{j}^{(0)}\right) \sum_{i=0}^{j}{ }_{j} \chi_{i}^{(k)} Q\left(\sqrt{\frac{i m_{u}^{(k)}+m_{u_{0}}}{2}}\right) \\
= & \underbrace{\sum_{j=2}^{d_{l}}\left(\lambda_{j}^{\prime} \pi_{j}^{(0)}\left(\varepsilon^{(k)}\right)^{j}\right)=p^{(k)}}_{\text {Unrecovered punctured symbols, } P_{e_{1}}^{(k)}} \\
& +\underbrace{\sum_{j=2}^{d_{l}} \lambda_{j}^{\prime} \pi_{j}^{(0)} \sum_{i=1}^{j} \chi_{j}^{(k)} Q\left(\sqrt{\frac{i m_{u}^{(k)}}{2}}\right)}_{\text {Recovered punctured symbols, } P_{e_{2}}^{(k)}} \\
& +\underbrace{\sum_{j=2}^{d_{l}} \lambda_{j}^{\prime}\left(1-\pi_{j}^{(0)}\right) \sum_{i=0}^{j} \chi_{i}^{(k)} Q\left(\sqrt{\frac{i m_{u}^{(k)}+m_{u_{0}}}{2}}\right)}_{\text {Unpunctured symbols, } P_{e_{3}}^{(k)}}
\end{aligned}
$$

where

$$
\sum_{j=2}^{d_{l}} \lambda_{j}^{\prime} \pi_{j}^{(0)}{ }_{j} \chi_{0}^{(k)} Q(0)=\sum_{j=2}^{d_{l}}\left(\lambda_{j}^{\prime} \pi_{j}^{(0)}\left(\varepsilon^{(k)}\right)^{j}\right) / 2
$$

In the error probability, the first term describes half of the unrecovered punctured fraction at the $k$ th iteration, the second term is the error probability due to the recovered punctured symbols, and the last term comes from the unpunctured symbols.
We may puncture only the parity part of a codeword. Thus, it is enough to recover the symbols in the unpunctured part, which happens when $P_{e_{3}}^{(k)} \rightarrow 0$. However, the following proposition tells that the first two terms also become zero when the last term does.

Proposition 1: If the unpunctured symbols converge to the corresponding transmitted symbols (correct symbols for short), the punctured symbols also converge to the correct symbols. Simply stated, $P_{e}^{(k)} \rightarrow 0 \Leftrightarrow P_{e}^{(k)} \rightarrow 0$.

Proof: Assume that the unpunctured symbols converge to the correct symbols, which means the last term in (14) converges to zero. Thus,

$$
\begin{aligned}
P_{e_{3}}^{(k)}= & \sum_{j=2}^{d_{l}} \lambda_{j}^{\prime}\left(1-\pi_{j}^{(0)}\right) \sum_{i=0}^{j}{ }_{j} \chi_{i}^{(k)} Q\left(\sqrt{\frac{i m_{u}^{(k)}+m_{u_{0}}}{2}}\right) \\
& \rightarrow 0 \text { implies } \\
& \sum_{j=2}^{d_{l}} \lambda_{j}^{\prime}\left(1-\pi_{j}^{(0)}\right) \sum_{i=1}^{j}{ }_{j} \chi_{i}^{(k)} Q\left(\sqrt{\frac{i m_{u}^{(k)}+m_{u_{0}}}{2}}\right) \\
& \rightarrow 0 \text { and } \\
& \sum_{j=2}^{d_{l}} \lambda_{j}^{\prime}\left(1-\pi_{j}^{(0)}\right)\left(\varepsilon^{(k-1)}\right)^{j-1} Q\left(\sqrt{\frac{m_{u_{0}}}{2}}\right) \rightarrow 0
\end{aligned}
$$

when $m_{u}^{(k)} \rightarrow \infty$ and $\varepsilon^{(k-1)} \rightarrow 0$, respectively. The recovered punctured symbols converge to the correct symbols because

$$
P_{e_{2}}^{(k)}=\sum_{i=1}^{j} j \chi_{i}^{(k)} Q\left(\sqrt{\frac{i m_{u}^{(k)}}{2}}\right) \rightarrow 0
$$

as $m_{u}^{(k)} \rightarrow \infty$. The fraction of the unrecovered punctured symbols $\left(P_{e_{1}}^{(k)}\right)$ also tends to zero due to $\varepsilon^{(k-1)} \rightarrow 0$ as $k \rightarrow \infty$.

The evolution of the puncturing fraction in (11) indicates that the residual puncturing fraction during iterations does not depend on SNR but only on a degree distribution pair $(\lambda(x), \rho(x))$ and a puncturing distribution $\pi^{(0)}(x)$, or in short, a three-tuple distribution $\left(\lambda(x), \rho(x), \pi^{(0)}(x)\right)$.

The following proposition gives a sufficient condition for the residual puncturing fraction to converge to zero.

Proposition 2: If $e^{(k)} \rightarrow 0$ (equivalently, $p^{(k)} \rightarrow 0$ ) as $k \rightarrow \infty$ for a puncturing distribution $\pi^{(0)}(x)$, then $e^{(k)} \rightarrow 0$ for any puncturing distribution $\omega^{(0)}(x)$ such that $\omega_{j}^{(0)} \leq \pi_{j}^{(0)}$.

Proof: We will prove this proposition by induction. $e^{(0)}(\omega) \leq$ $e^{(0)}(\pi)$ since

$$
\begin{aligned}
e^{(0)}(\omega) & =\sum_{j=2}^{d_{l}} \omega_{j}^{(0)} \lambda_{j} \\
e^{(0)}(\pi) & =\sum_{j=2}^{d_{l}} \pi_{j}^{(0)} \lambda_{j}
\end{aligned}
$$

and

$$
\omega_{j}^{(0)} \lambda_{j} \leq \pi_{j}^{(0)} \lambda_{j}
$$

$$
m_{u}^{(k)}=\sum_{s=2}^{d_{r}} \rho_{s} \phi^{-1}\left(1-\frac{1}{\left(1-e^{(k)}\right)^{s-1}}\left[1-\sum_{j=2}^{d_{l}}\left\{\lambda_{j}^{\pi} \sum_{i=0}^{j-1}(j-1) \chi_{i}^{(k)} \phi\left(i m_{u}^{(k-1)}\right)+\lambda_{j}^{(1-\pi)} \sum_{i=0}^{j-1}(j-1) \chi_{i}^{(k)} \phi\left(i m_{u}^{(k-1)}+m_{u_{0}}\right)\right\}\right]^{s-1}\right)
$$




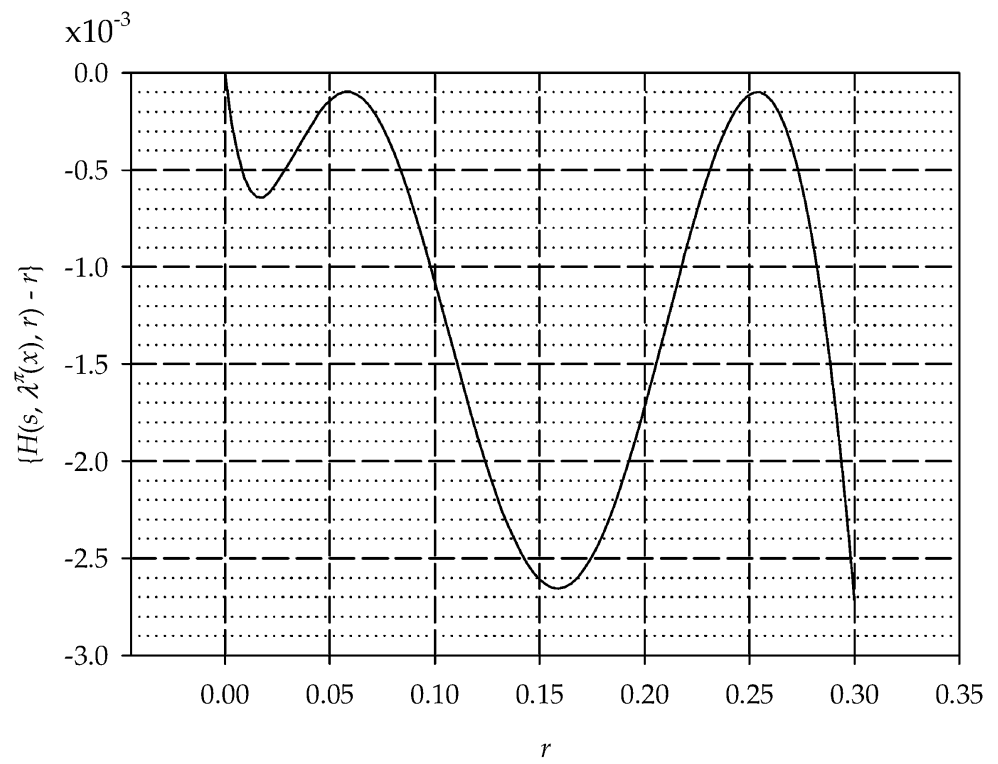

Fig. 5. $\left\{H\left(s, \lambda^{\pi}(x), r\right)-r\right\}$ and $\min \left|H\left(s, \lambda^{\pi}(x), r\right)-r\right|=10^{-4}$ for $s=2.76700$ and $p^{(0)}=0.25$.

Suppose $e^{(k)}(\omega) \leq e^{(k)}(\pi)$ for $k>0$, then $e^{(k+1)}(\omega) \leq e^{(k+1)}(\pi)$ since

$$
\begin{aligned}
e^{(k+1)}(\omega) & =\sum_{j=2}^{d_{l}} \omega_{j}^{(0)} \lambda_{j}\left(1-\rho\left(1-e^{(k)}(\omega)\right)\right) \\
& \leq \sum_{j=2}^{d_{l}} \omega_{j}^{(0)} \lambda_{j}\left(1-\rho\left(1-e^{(k)}(\pi)\right)\right) \\
& \leq \sum_{j=2}^{d_{l}} \pi_{j}^{(0)} \lambda_{j}\left(1-\rho\left(1-e^{(k)}(\pi)\right)\right) \\
& =e^{(k+1)}(\pi) .
\end{aligned}
$$

The relation between (a) and (b) holds since $0<e^{(k)}(\omega) \leq e^{(k)}(\pi)$. The inequality between (b) and (c) holds due to $\omega_{j}^{(0)} \leq \pi_{j}^{(0)}$.

It should be noted that Proposition 2 does not tell the convergence with respect to a puncturing fraction $p^{(0)}$ since $p^{(0)}(\omega) \leq p^{(0)}(\pi)$ does not imply that $\omega_{j} \leq \pi_{j}$, for all $j$.

In the case when $e^{(k)}\left(\varepsilon^{(k)}\right)$ converges to zero, we can approximate ${ }_{j} \chi_{i}$ as $\delta_{j i}$, which simplifies the recursive (13) as

$$
\begin{array}{r}
m_{u}^{(k)}=\sum_{s=2}^{d_{r}} \rho_{s} \phi^{-1}\left(1-\left[1-\sum_{j=2}^{d_{l}}\left\{\lambda_{j}^{\pi} \phi\left((j-1) m_{u}^{(k-1)}\right)\right.\right.\right. \\
\left.\left.\left.+\lambda_{j}^{(1-\pi)} \phi\left((j-1) m_{u}^{(k-1)}+m_{u_{0}}\right)\right\}\right]^{s-1}\right) .
\end{array}
$$

We call this simplified equation a steady-state equation.

For error-free decoding $\left(P_{e}^{(k)} \rightarrow 0\right.$ in (14)), the updated mean grows monotonically to infinity, which means $m_{u}^{(k)}<m_{u}^{(k+1)}$ for any $k \geq 0$ and $m_{u}^{(k)} \rightarrow \infty$ as $k \rightarrow \infty$. This inequality $\left(m_{u}^{(k)}<m_{u}^{(k+1)}\right)$ can be equivalently expressed as

$$
\begin{aligned}
r & >\sum_{j=2}^{d_{l}} \lambda_{j}^{\pi} h_{j}(0, r)+\lambda_{j}^{(1-\pi)} h_{j}\left(m_{u_{0}}, r\right) \\
& =h\left(0, \lambda^{\pi}(x), r\right)+h\left(m_{u_{0}}, \lambda^{(1-\pi)}(x), r\right) \\
& =H\left(m_{u_{0}}, \lambda^{\pi}(x), r\right), \quad \forall r \in\left(0, \phi\left(m_{u_{0}}\right)\right]
\end{aligned}
$$

where $h_{j}(s, r)$ is defined in Section II

$$
h\left(s, \lambda^{q}(x), r\right)=\sum_{j=2}^{d_{l}} \lambda_{j}^{q} h_{j}(s, r)=E_{\lambda_{j}^{q}}\left[h_{j}(s, r)\right]
$$

$q$ is either $\pi$ or $1-\pi$, and

$$
H\left(s, \lambda^{\pi}(x), r\right)=h\left(0, \lambda^{\pi}(x), r\right)+h\left(s, \lambda^{(1-\pi)}(x), r\right) .
$$

The inequality in (16) gives us an insight into a design rule for good puncturing distributions. We can get $h\left(m_{u_{0}}, \lambda^{(1-\pi)}(x), r\right)$ and $h\left(m_{u_{0}}, \lambda^{\pi}(x), r\right)$ by averaging $h_{j}\left(m_{u_{0}}, r\right)$ and $h_{j}(0, r)$ with $\lambda_{j}^{(1-\pi)}$ and $\lambda_{j}^{\pi}$, respectively. Finally, $H\left(m_{u_{0}}, \lambda^{\pi}(x), r\right)$ is calculated by summing $h\left(m_{u_{0}}, \lambda^{(1-\pi)}(x), r\right)$ and $h\left(m_{u_{0}}, \lambda^{\pi}(x), r\right)$. To satisfy the inequality, $\left\{H\left(m_{u_{0}}, \lambda^{\pi}(x), r\right)-r\right\}$ must be less than zero when $0<r \leq \phi\left(m_{u_{0}}\right)$. When summing $h_{j}\left(m_{u_{0}}, r\right)$ and $h_{j}(0, r)$, we have freedom to weight $h_{j}\left(m_{u_{0}}, r\right)$ and $h_{j}(0, r)$ by optimizing puncturing proportions, $\pi_{j}^{(0)}$, s, to maximize $p^{(0)}$ for a given $m_{u_{0}}$, which is equivalent to choosing $\lambda_{j}^{\pi}$ and $\lambda_{j}^{(1-\pi)}$ to minimize $m_{u_{0}}$ for a given $p^{(0)}$.

In Fig. 5, we can see that $\left\{H\left(s, \lambda^{\pi}(x), r\right)-r\right\}$ barely averts touching zero, which is a fixed point of the recursive equation (in this example, min $\left.\left|H\left(s, \lambda^{\pi}(x), r\right)-r\right|=10^{-4}\right)$. A smaller gap between $\left\{H\left(s, \lambda^{\pi}(x), r\right)-r\right\}$ and the $r$ axis makes a decoder iterate more for achieving error-free decoding, although the SNR threshold may be decreased.

We can rewrite (16) as

$$
\underbrace{\sum_{j=2}^{d_{l}} \lambda_{j} \pi_{j}^{(0)}\left(h_{j}(0, r)-h_{j}\left(m_{u_{0}}, r\right)\right)}_{\text {a linear combination of } \pi_{j}^{(0) \text { s }_{\mathrm{s}}}}+\underbrace{\sum_{j=2}^{d_{l}} \lambda_{j} h_{j}\left(m_{u_{0}}, r\right)}_{\text {a constant }}<r
$$

and the inequality contains a linear combination of $\pi_{j}^{(0)}$ 's. Thus, we can design a puncturing distribution for a given degree distribution pair with linear programming, which is formulated in Fig. 6.

To use the design rule, the designed puncturing distribution must satisfy the required condition for the steady-state equation, which is $e^{(k)} \rightarrow 0$ as $k \rightarrow \infty$. If we design $\pi^{(0)}(x)$ with the design rule in 


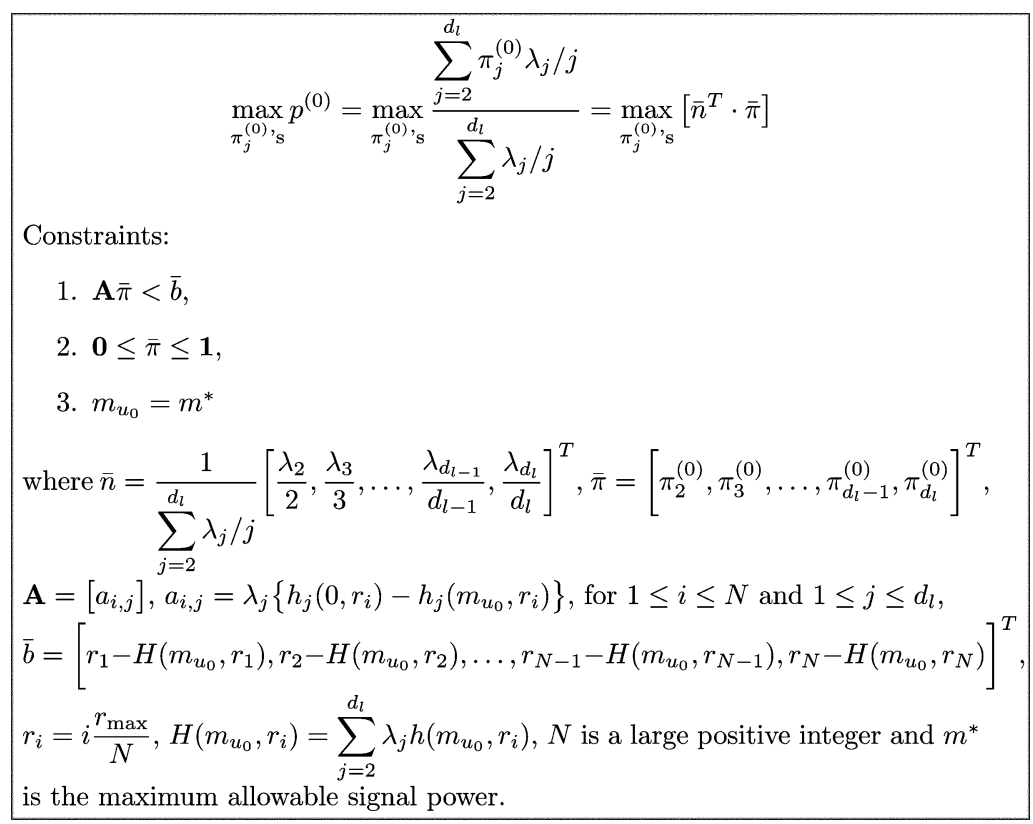

Fig. 6. Proposed design rule with linear programming.

Fig. 6, we can say that $e^{(k)} \rightarrow 0$ as $k \rightarrow \infty$ when a right degree distribution has the concentrated form [13]. We shall prove the claim in a heuristic way by showing that a designed puncturing distribution $\pi^{(0)}(x)$ satisfies the following inequality:

$$
\begin{aligned}
& \sum_{j=2}^{d_{l}} \lambda_{j} \pi_{j}^{(0)}\left(1-\sum_{i=2}^{d_{r}} \rho_{i}(1-r)^{i-1}\right)^{j-1} \\
& \quad=\sum_{j=2}^{d_{l}} \lambda_{j} \pi_{j}^{(0)} e_{j}(r) \\
& \quad \leq \sum_{j=2}^{d_{l}}\left\{\lambda_{j} \pi_{j}^{(0)} h_{j}(0, r)+\lambda_{j}\left(1-\pi_{j}^{(0)}\right) h_{j}\left(m_{u_{0}}, r\right)\right\}<r
\end{aligned}
$$

A sufficient condition is $e_{j}(r) \leq h_{j}(0, r)$ since

$$
\begin{aligned}
\sum_{j=2}^{d_{l}} \lambda_{j} \pi_{j}^{(0)} h_{j} & (0, r) \\
\leq & \sum_{j=2}^{d_{l}}\left\{\lambda_{j} \pi_{j}^{(0)} h_{j}(0, r)+\lambda_{j}\left(1-\pi_{j}^{(0)}\right) h_{j}\left(m_{u_{0}}, r\right)\right\}
\end{aligned}
$$

where $0 \leq h_{j}\left(m_{u_{0}}, r\right) \leq 1$ and $0 \leq \pi_{j}^{(0)} \leq 1$. In most practical cases, right degree distributions for AWGN channels have the concentrated form $\rho(x)=\rho x^{d_{r}-2}+(1-\rho) x^{d_{r}-1}$ [13], and $d_{r}$ is large enough for the approximation

$$
\begin{aligned}
\sum_{i=2}^{d_{r}} \rho_{i} \phi^{-1} & \left(1-(1-r)^{i-1}\right) \\
& \approx \phi^{-1}\left(\sum_{i=2}^{d_{r}} \rho_{i}\left(1-(1-r)^{i-1}\right)\right) \\
& =\phi^{-1}\left(1-\sum_{i=2}^{d_{r}} \rho_{i}(1-r)^{i-1}\right)
\end{aligned}
$$

since $(1-r)^{d_{r}-2}-(1-r)^{d_{r}-1}=r(1-r)^{d_{l}-2} \ll 1$ and $\phi^{-1}(\cdot)$ can be linearized between $1-(1-r)^{d_{r}-2}$ and $1-(1-r)^{d_{r}-1}$. Thus, we can approximate $h_{j}(0, r)$ as

$$
\begin{aligned}
h_{j}(0, r) & =\phi\left((j-1) \sum_{i=2}^{d_{r}} \rho_{i} \phi^{-1}\left(1-(1-r)^{i-1}\right)\right) \\
& \approx \phi\left((j-1) \phi^{-1}\left(1-\sum_{i=2}^{d_{r}} \rho_{i}(1-r)^{i-1}\right)\right)
\end{aligned}
$$

and rewrite $e_{j}(r)$ as

$$
e_{j}(r)=\phi\left(\phi^{-1}\left(\left[1-\sum_{i=2}^{d_{r}} \rho_{i}(1-r)^{i-1}\right]^{j-1}\right)\right) .
$$

If $x^{n}=\phi\left(\phi^{-1}\left(x^{n}\right)\right) \leq \phi\left(n \phi^{-1}(x)\right)$ for $0 \leq x \leq 1$ and $n \geq 1$, we can say that $e_{j}(r) \leq h_{j}(0, r)$. In the following proposition, we show that the inequality $\phi\left(\phi^{-1}\left(x^{n}\right)\right) \leq \phi\left(n \phi^{-1}(x)\right)$ holds by using the convexity ${ }^{1}$ of $\phi(x)$.

Proposition 3: $x^{n}=\phi\left(\phi^{-1}\left(x^{n}\right)\right) \leq \phi\left(n \phi^{-1}(x)\right)$ for $0 \leq x \leq 1$ and $n \geq 1$.

Proof: We will prove this proposition by induction. The equality holds at $x=1$ and 0 for any $n \geq 1$ since $\phi^{-1}(1)=0, \phi(0)=1$, $\phi^{-1}(0)=\infty$, and $\phi(\infty)=0$. For $n=1, x=\phi\left(\phi^{-1}(x)\right)$. Suppose $x^{n} \leq \phi\left(n \phi^{-1}(x)\right)$ for $n>1$, then

$$
x^{n+1}=x \cdot x^{n} \leq x \cdot \phi\left(n \phi^{-1}(x)\right) .
$$

Since $\phi(x)$ is a continuous convex function of $x$, the following inequality holds [14]:

$$
\frac{\phi(x)-\phi(w)}{x-w} \leq \frac{\phi(z)-\phi(y)}{z-y}
$$

${ }^{1}$ Since $\phi(\boldsymbol{x})$ seems to be mathematically intractable, we numerically verified that the second derivative of $\boldsymbol{\phi}(\boldsymbol{x})$ is greater than or equal to zero, which is the necessary and sufficient condition for convexity. 


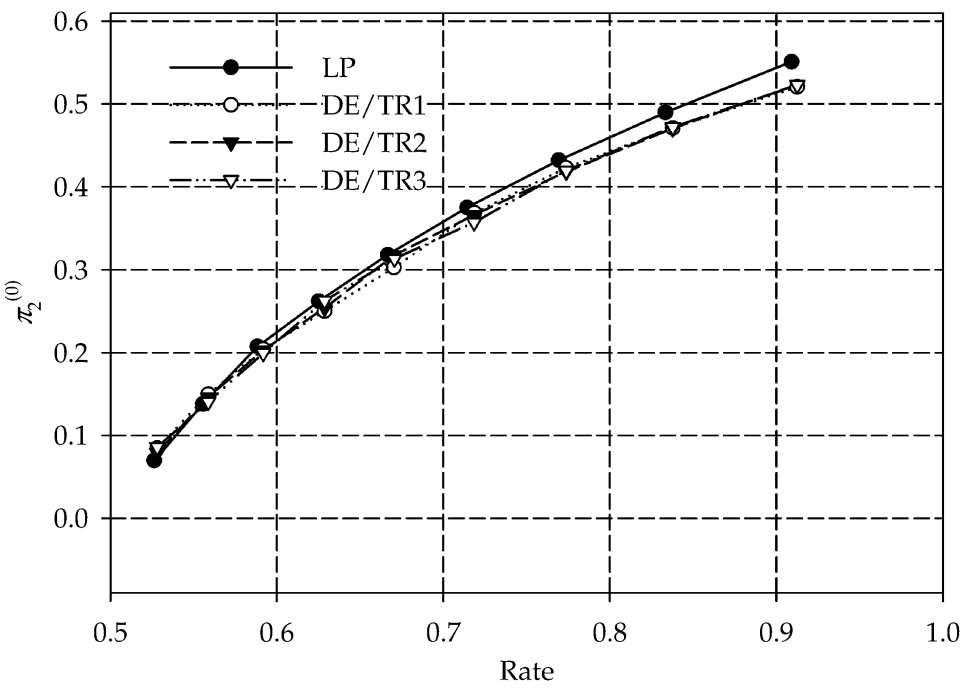

Fig. 7. $\pi_{2}^{(0)}$,s designed with linear programming and differential evolution (DE) over $\bigcup^{4}(\mathrm{TR} 1)$ and $\mathrm{DE}$ with initial values from linear programming perturbed by uniform (TR2) and Gaussian (TR3) for a degree distribution pair, $\lambda(x)=0.25105 x+0.30938 x^{2}+0.00104 x^{3}+0.43853 x^{9}$ and $\rho(x)=0.63676 x^{6}+$ $0.36324 x^{7}$.

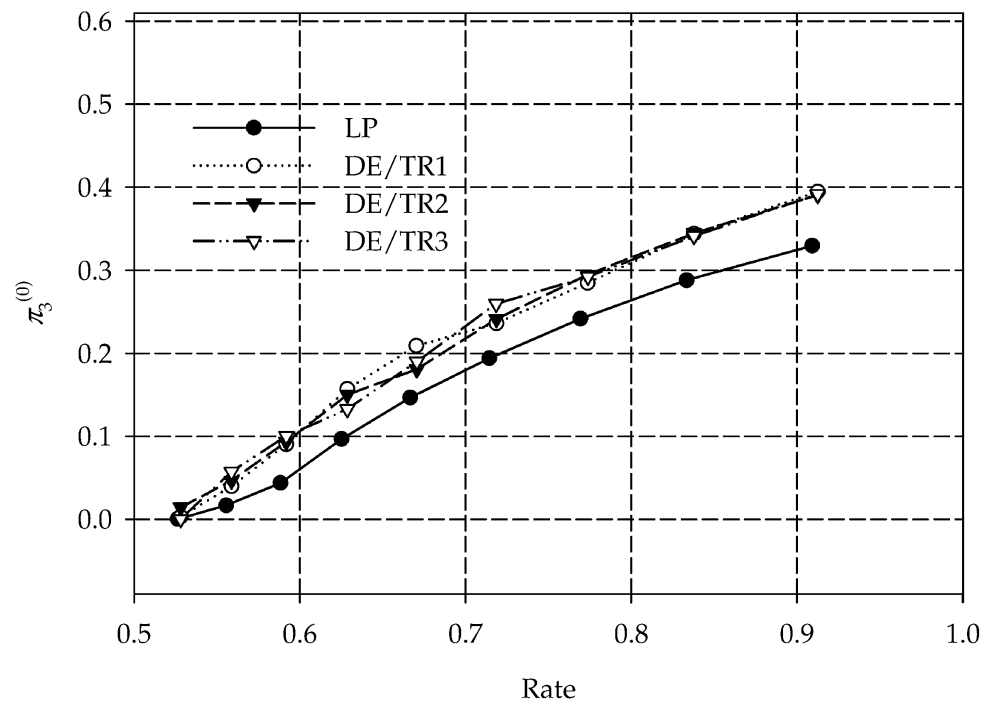

Fig. 8. $\pi_{3}^{(0)}$, s designed with linear programming and DE over $U^{4}$ (TR1) and DE with initial values from linear programming perturbed by uniform (TR2) and Gaussian (TR3) for a degree distribution pair, $\lambda(x)=0.25105 x+0.30938 x^{2}+0.00104 x^{3}+0.43853 x^{9}$ and $\rho(x)=0.63676 x^{6}+0.36324 x^{7}$.

for $w<x<y<z$. By replacing $w, x, y$, and $z$ with $0, \phi^{-1}(x)$, $n \phi^{-1}(x)$, and $(n+1) \phi^{-1}(x)$, respectively, we can rewrite the inequality as

$$
\begin{aligned}
\frac{\phi\left(\phi^{-1}(x)\right)-\phi(0)}{\phi^{-1}(x)-0} & =\frac{\phi\left(\phi^{-1}(x)\right)-1}{\phi^{-1}(x)} \\
& \leq \frac{\phi\left((n+1) \phi^{-1}(x)\right)-\phi\left(n \phi^{-1}(x)\right)}{(n+1) \phi^{-1}(x)-n \phi^{-1}(x)} \\
& =\frac{\phi\left((n+1) \phi^{-1}(x)\right)-\phi\left(n \phi^{-1}(x)\right)}{\phi^{-1}(x)} \\
& \leq \frac{\phi\left((n+1) \phi^{-1}(x)\right)-\phi\left(n \phi^{-1}(x)\right)}{\phi^{-1}(x) \phi\left(n \phi^{-1}(x)\right)} \\
& =\frac{\phi\left((n+1) \phi^{-1}(x)\right) / \phi\left(n \phi^{-1}(x)\right)-1}{\phi^{-1}(x)}
\end{aligned}
$$

where $x \in[0,1), \phi(0)=1$ in (a), and $0<\phi(a) \leq 1$ for any $a \geq 0$ in (b). The relation between (a) and (c) gives us

$$
x^{n+1} \leq x \phi\left(n \phi^{-1}(x)\right) \leq \phi\left((n+1) \phi^{-1}(x)\right)
$$

for any $n \geq 1$ and $x \in[0,1]$.

\section{DESIGN OF PUNCTURING Distributions FOR BASE $R=1 / 2$ CODE}

In this section, we design puncturing distributions with two good edge degree distribution pairs from [7] and [11] for 1/2-rate LDPC codes that have the maximum variable degrees of 10 and 20 , respectively. The design goal is either to maximize the puncturing fraction $p^{(0)}$ for a given $E_{b} / N_{0}$ or to minimize the $E_{b} / N_{0}$ threshold for a given puncturing fraction.

We minimize the $E_{b} / N_{0}$ thresholds with linear programming for puncturing fractions $p^{(0)}$ 's ranging from 0.05 to 0.45 in increments of 0.05 to achieve the rate $R$ from 0.5263 to 0.9091 with the degree distribution pair in [7]

$$
\lambda(x)=0.25105 x+0.30938 x^{2}+0.00104 x^{3}+0.43853 x^{9}
$$

and

$$
\rho(x)=0.63676 x^{6}+0.36324 x^{7} .
$$




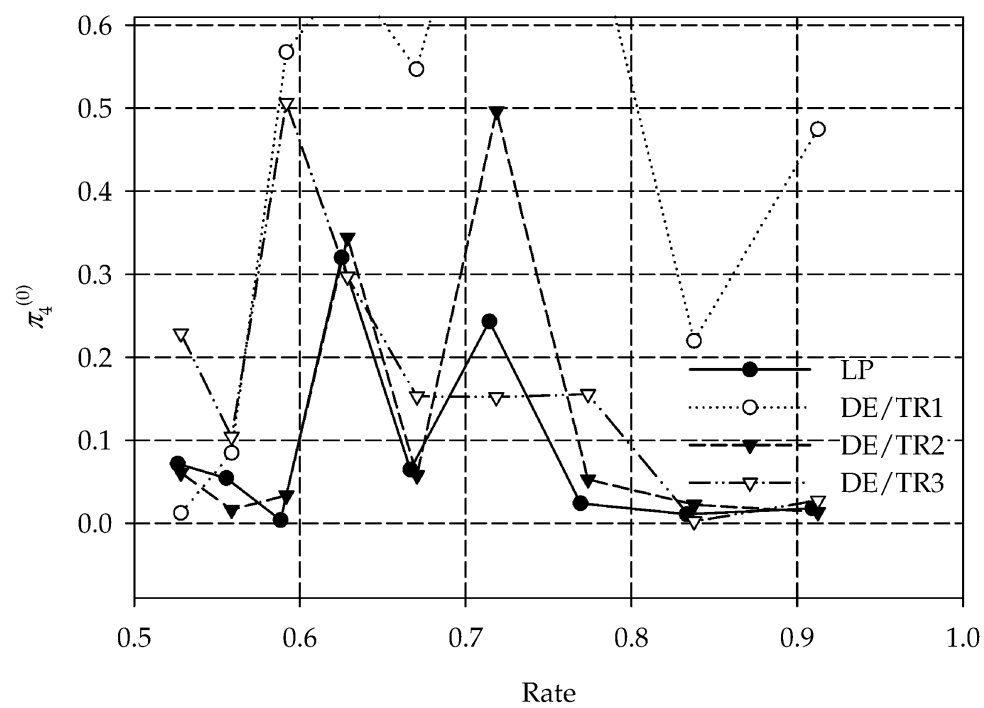

Fig. 9. $\pi_{4}^{(0)}$,s designed with linear programming and DE over $\cup^{4}$ (TR1) and DE with initial values from linear programming perturbed by uniform (TR2) and Gaussian (TR3) for a degree distribution pair, $\lambda(x)=0.25105 x+0.30938 x^{2}+0.00104 x^{3}+0.43853 x^{9}$ and $\rho(x)=0.63676 x^{6}+0.36324 x^{7}$.

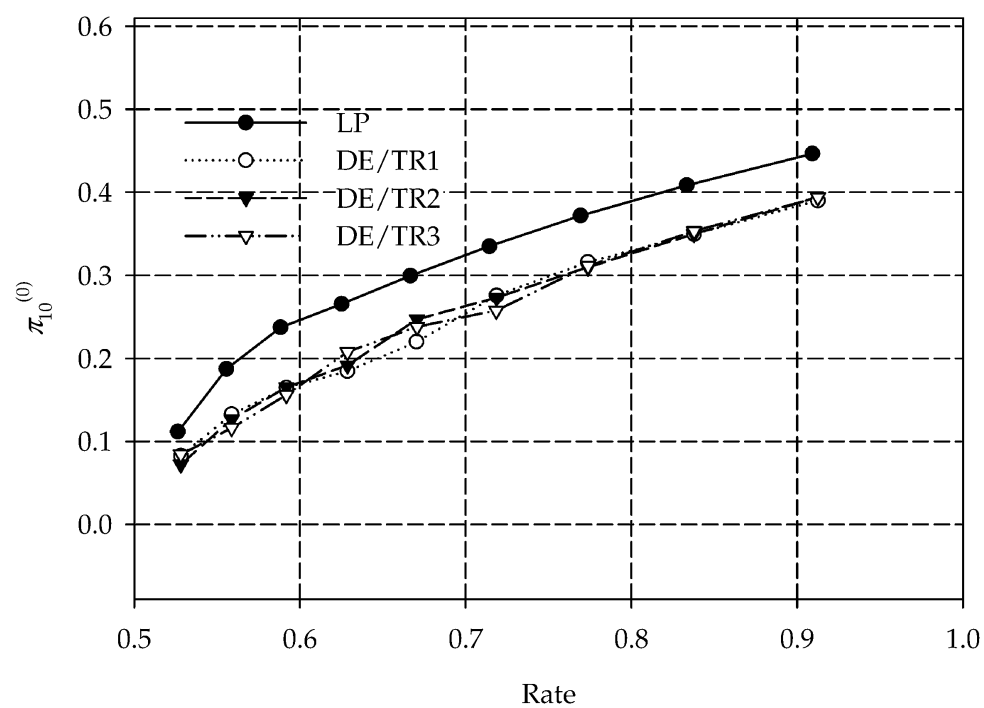

Fig. 10. $\pi_{10}^{(0)}$ 's designed with linear programming and DE over $\mathrm{U}^{4}$ (TR1) and DE with initial values from linear programming perturbed by uniform (TR2) and Gaussian (TR3) for a degree distribution pair, $\lambda(x)=0.25105 x+0.30938 x^{2}+0.00104 x^{3}+0.43853 x^{9}$ and $\rho(x)=0.63676 x^{6}+0.36324 x^{7}$.

In Figs. 7-10, we depict the designed puncturing proportions, $\pi_{j}^{(0)}$, s (denoted as LP) obtained by the design rule in Fig. 6, all but one of which increase monotonically with the growing rate, indicating that the codes can be punctured in a rate-compatible fashion. The abrupt changes of $\pi_{4}^{(0)}$ are caused by the small magnitude of $\lambda_{4}$, which means the size of $G_{4}$ is so small that the puncturing of symbols in $G_{4}$ does not have much influence on the overall performance. Thus, the numerical routine for the linear programming gives us the meaningless values of $\pi_{4}^{(0)}$ s. The puncturing proportions obtained from the linear programming are listed in Table I.

We can design rate-compatible puncturing distributions whose puncturing proportions, $\pi_{j}^{(0)}$, s, must be increasing functions by adding an additional constraint, $\pi_{4}^{(0)}\left(R_{2}\right) \geq \pi_{4}^{(0)}\left(R_{1}\right)$, where $R_{2}$ and $R_{1}$ are rates, and $R_{2} \geq R_{1}$. However, the constraint does not change the results of other puncturing proportions, $\pi_{j}^{(0)}$, s, for $j \neq 4$ since $\pi_{4}^{(0)}$ does not have meaningful influence on the overall performance.

To verify the analytic results and the design rule with linear programming, we also design the puncturing distributions with the DDE [11] technique and the differential evolution (DE) [15] technique that searches for an optimum vector with a combination of a genetic and a hill climbing algorithms. The results are listed in Table II. The DDE algorithm models the message-passing decoder more accurately, although the algorithm lacks analytic insight and requires more computation. We compare the results from linear programming (denoted as LP) with the ones from DE in Figs. 7-10. The optimizations with DE proceed in three different ways to avoid possible local minima. In the first trial (denoted as TR1), we search for the puncturing distribution over the $U^{4}$ space without initial values where $U=[0,1]$, and $U^{4}$ is the domain of four independent variables $\left(\pi_{2}^{(0)}, \pi_{3}^{(0)}, \pi_{4}^{(0)}\right.$, and $\left.\pi_{10}^{(0)}\right)$. In the second and the third trials (denoted as TR2 and TR3), we use the puncturing proportions $\pi_{j}^{(0)}$, s obtained from LP as initial values that are perturbed with Gaussian and uniform noise, respectively. The comparisons show that there is no distinctive difference among the results from the three different trials with DE. However, we can see some discrepancies between the results from LP and the ones from DE, although they have the same trends with respect to the rate. The discrepancies are more distinctive at higher degrees, which comes from the fact that node distribution is inversely proportional to the degree in (1), $\left(\lambda_{j}^{\prime}=\alpha \lambda_{j} / j\right.$ 
TABLE I

PUNCTURING PROPORTIONS, $\pi_{j}^{(0)}$ 's, FOR $\lambda(x)=0.25105 x+0.30938 x^{2}+0.00104 x^{3}+0.43853 x^{9}$ AND $\rho(x)=0.63676 x^{6}+0.36324 x^{7}$ AT THE OVERALl PUNCTURING PRoBabILITIES OF $0.00,0.05,0.10,0.15,0.20,0.25,0.30,0.35,0.40$, AND 0.45 With LiNEAR PROGRAMMING

\begin{tabular}{|c|c|c|c|c|c|c|c|c|c|c|}
\hline$\sigma$ & 0.95574 & 0.91582 & 0.87463 & 0.83283 & 0.78866 & 0.74095 & 0.68875 & 0.63009 & 0.56086 & 0.46754 \\
\hline \hline$\pi_{2}^{(0)}$ & 0.00000 & 0.06944 & 0.14806 & 0.20729 & 0.26188 & 0.31805 & 0.37500 & 0.43221 & 0.48985 & 0.55105 \\
\hline$\pi_{3}^{(0)}$ & 0.00000 & 0.00004 & 0.00021 & 0.04350 & 0.09652 & 0.14650 & 0.19395 & 0.24140 & 0.28801 & 0.32952 \\
\hline$\pi_{4}^{(0)}$ & 0.00000 & 0.07155 & 0.05424 & 0.00373 & 0.32006 & 0.06447 & 0.24312 & 0.02393 & 0.01062 & 0.01762 \\
\hline$\pi_{10}^{(0)}$ & 0.00000 & 0.11172 & 0.19739 & 0.23732 & 0.26550 & 0.29973 & 0.33503 & 0.37200 & 0.40846 & 0.44665 \\
\hline \hline$p^{(0)}$ & 0.00000 & 0.05000 & 0.10000 & 0.15000 & 0.20000 & 0.25000 & 0.30000 & 0.35000 & 0.40000 & 0.45000 \\
\hline
\end{tabular}

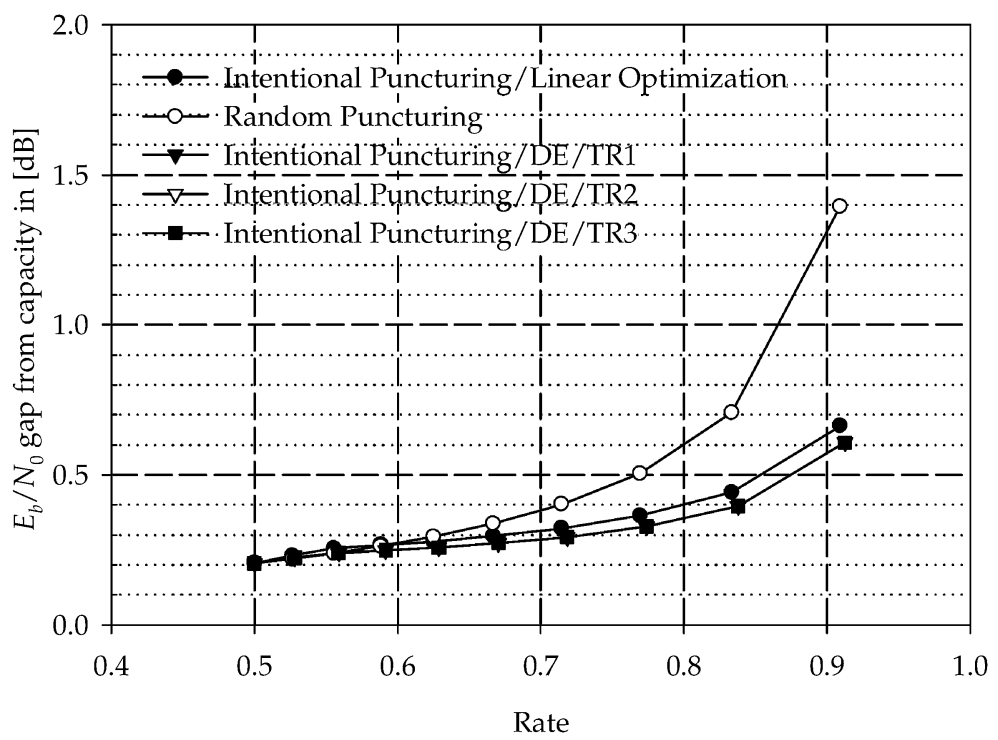

Fig. 11. Gaps between asymptotic $E_{b} / N_{0}$ 's and the capacity of BPSK signal with respect to the rates for a degree distribution pair, $\lambda(x)=0.25105 x+$ $0.30938 x^{2}+0.00104 x^{3}+0.43853 x^{9}$ and $\rho(x)=0.63676 x^{6}+0.36324 x^{7}$.

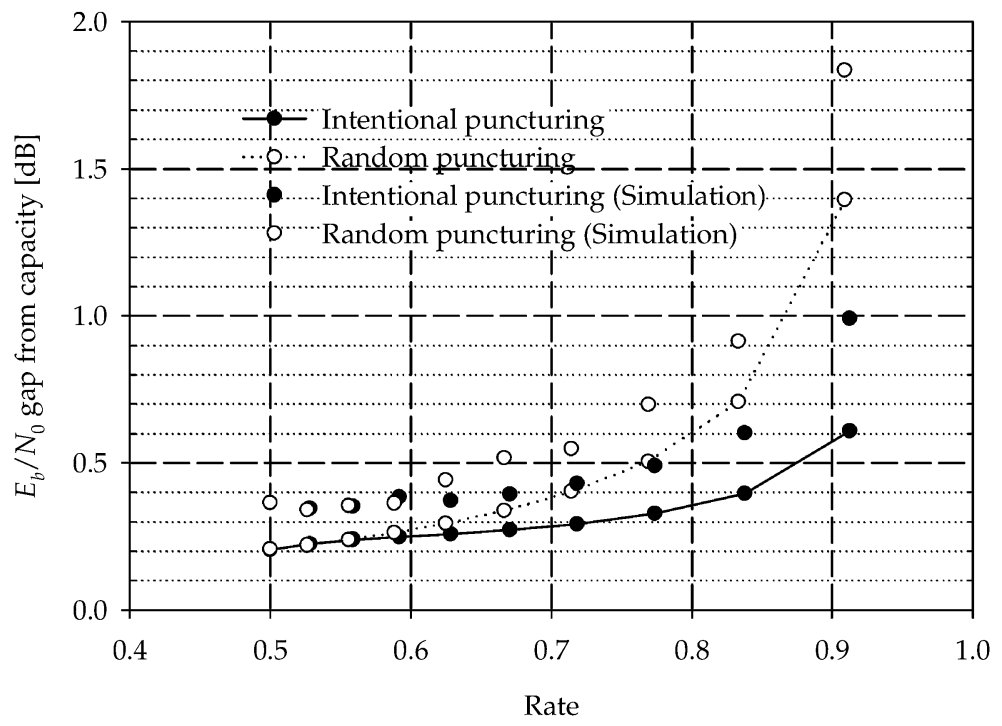

Fig. 12. Gaps between asymptotic $E_{b} / N_{0}$ 's and the capacity of BPSK signal with respect to the rates for a degree distribution pair, $\lambda(x)=0.25105 x+$ $0.30938 x^{2}+0.00104 x^{3}+0.43853 x^{9}$ and $\rho(x)=0.63676 x^{6}+0.36324 x^{7}$.

and $\alpha>0$ ). Thus, the lowest degree puncturing proportion $\pi_{2}^{(0)}$ is the most important which has a good match with the results from DE in Fig. 7.

For comparison, we also design punctured LDPC codes with the puncturing distribution

$$
\pi^{(0)}(x)=\sum_{j} p^{(0)} x^{j-1}
$$

The proposed and the uniform puncturing schemes are called intentional puncturing and random puncturing, respectively, when we compare them. Elsewhere, we will call the proposed puncturing scheme simply puncturing.

We compute the gaps between the asymptotic $E_{b} / N_{0}$ thresholds and the capacity of the binary-phase-shift keying (BPSK) signal with respect to the rate in Fig. 11. The asymptotic $E_{b} / N_{0}$ thresholds are 
TABLE II

PUNCTURING PROPORTIONS, $\pi_{j}^{(0)}$, S, FOR $\lambda(x)=0.25105 x+0.30938 x^{2}+0.00104 x^{3}+0.43853 x^{9}$ AND $\rho(x)=0.63676 x^{6}+0.36324 x^{7}$ AT $\sigma$ 's USED IN LINEAR PROGRAMMING

\begin{tabular}{|c|c|c|c|c|c|c|c|c|c|c|}
\hline$\sigma$ & 0.95574 & 0.91582 & 0.87463 & 0.83283 & 0.78866 & 0.74095 & 0.68875 & 0.63009 & 0.56086 & 0.46754 \\
\hline \hline$\pi_{2}^{(0)}$ & 0.00000 & 0.07886 & 0.14628 & 0.20276 & 0.25381 & 0.31767 & 0.36624 & 0.41838 & 0.47074 & 0.52325 \\
\hline$\pi_{3}^{(0)}$ & 0.00000 & 0.01405 & 0.04641 & 0.09305 & 0.15000 & 0.18079 & 0.24119 & 0.29462 & 0.34447 & 0.39074 \\
\hline$\pi_{4}^{(0)}$ & 0.00000 & 0.06081 & 0.01616 & 0.03356 & 0.34406 & 0.05752 & 0.49649 & 0.05265 & 0.02227 & 0.01324 \\
\hline$\pi_{10}^{(0)}$ & 0.00000 & 0.07206 & 0.19149 & 0.16504 & 0.19149 & 0.24692 & 0.27318 & 0.30975 & 0.34997 & 0.39436 \\
\hline \hline$p^{(0)}$ & 0.00000 & 0.05324 & 0.10520 & 0.15505 & 0.20462 & 0.25430 & 0.30412 & 0.35378 & 0.40316 & 0.45194 \\
\hline
\end{tabular}

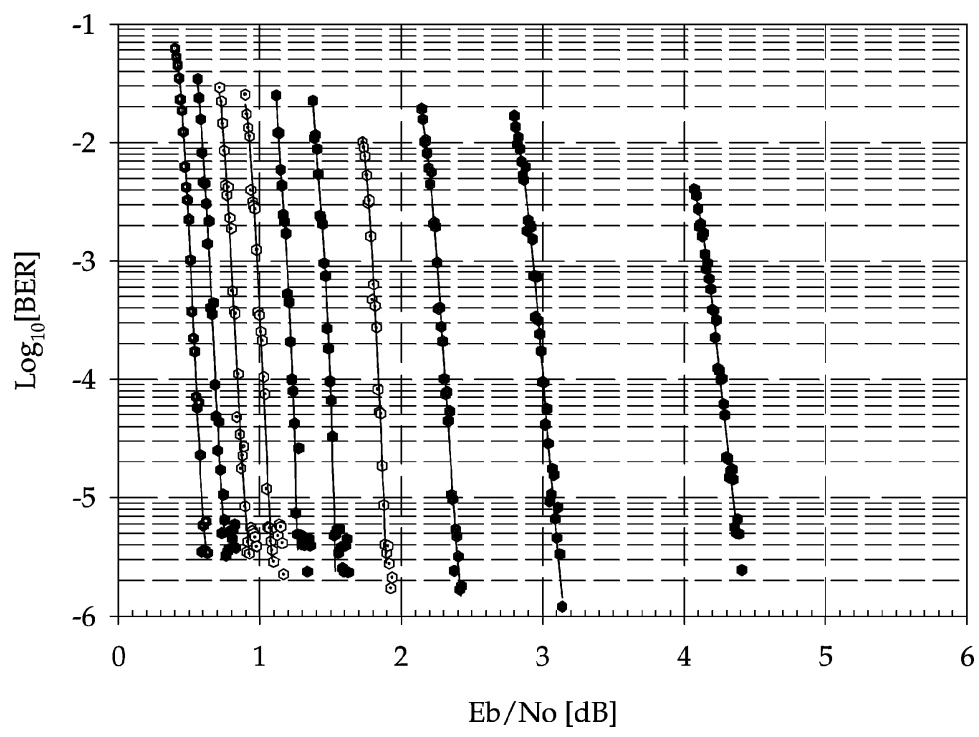

Fig. 13. BERs of the LDPC code $\left(\operatorname{lambda}(x)=0.25105 x+0.30938 x^{2}+0.00104 x^{3}+0.43853 x^{9}\right.$ and $\left.\rho(x)=0.63676 x^{6}+0.36324 x^{7}\right)$ with the puncturing fractions $p^{(0)}=0.0000,0.05324,0.10520,0.15505,0.20462,0.25430,0.30412,035378,0.40316$, and 0.45194 (left to right).

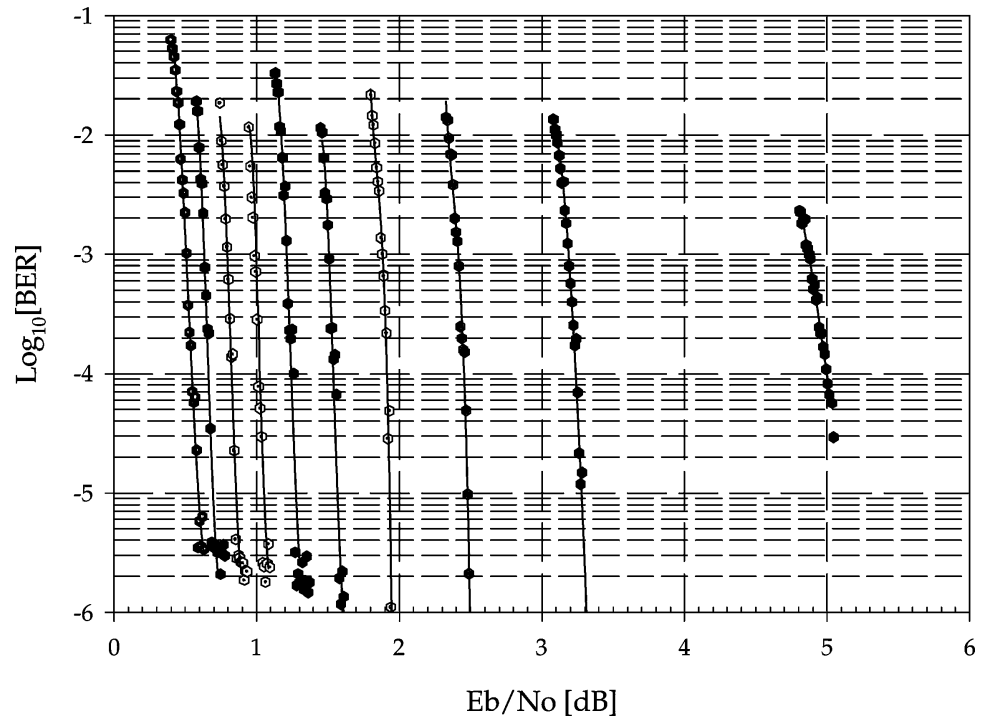

Fig. 14. BERs of the LDPC code $\left(\lambda(x)=0.25105 x+0.30938 x^{2}+0.00104 x^{3}+0.43853 x^{9}\right.$ and $\left.\rho(x)=0.63676 x^{6}+0.36324 x^{7}\right)$ with the random puncturing fractions, $p^{(0)}=0.00000,0.05000,0.10000,0.15000,0.20000,0.25000,0.30000,0.35000,0.40000$, and 0.45000 (left to right).

evaluated using DDE. We can notice that there is less than 0.1-dB difference between the thresholds of the punctured LDPC code with the puncturing distributions from LP and the ones from DE. Although the difference between the LDPC code with puncturing distributions from LP and DE is relatively small, we are more interested in the ultimate performance of the punctured LDPC codes. Thus, we describe the thresholds of the punctured LDPC codes with the puncturing distributions from DE. The results show that the base code has a 0.2-dB gap from the capacity and an additional $0.1-\mathrm{dB}$ gap permits us to vary the rate up to 0.72 . The changes in rate up to 0.84 and 0.92 are obtained at the expense of $0.2-$ and $0.4-\mathrm{dB}$ additional gaps, respectively.

To verify the design rule, we implement the LDPC code and evaluate required $E_{b} / N_{0}$ 's for a bit-error rate (BER) of $10^{-4}$ of the intentional and random puncturing schemes. The implemented LDPC code has a block length of 131072 , a rate of 0.5 , and no 4 cycle loops in the parity-check matrix. For the rate-compatibility, the number of punc- 
TABLE III

PUNCTURING PROPORTIONS $\pi_{j}^{(0)}$ 's FOR $\lambda(x)=0.23403 x+0.21242 x^{2}+0.14690 x^{5}+0.10284 x^{6}+0.30381 x^{19}$ AND $\rho(x)=0.71875 x^{7}+0.28125 x^{8}$ AT THE OVERALl PUNCTURING PROBABILITIES OF 0.00, 0.05, 0.10, 0.15, 0.20, 0.25, 0.30, 0.35, 0.40 AND 0.45 WITH LINEAR PROGRAMMING

\begin{tabular}{|c|c|c|c|c|c|c|c|c|c|c|}
\hline$\sigma$ & 0.96612 & 0.91177 & 0.88555 & 0.84154 & 0.79457 & 0.74385 & 0.68804 & 0.62472 & 0.54815 & 0.43501 \\
\hline \hline$\pi_{2}^{(0)}$ & 0.00000 & 0.05264 & 0.10204 & 0.16435 & 0.22641 & 0.28807 & 0.34894 & 0.41032 & 0.46910 & 0.53375 \\
\hline$\pi_{3}^{(0)}$ & 0.00000 & 0.00867 & 0.06497 & 0.10882 & 0.14149 & 0.17505 & 0.21015 & 0.24330 & 0.28408 & 0.30992 \\
\hline$\pi_{6}^{(0)}$ & 0.00000 & 0.00028 & 0.06549 & 0.12196 & 0.21268 & 0.30055 & 0.38902 & 0.48388 & 0.56178 & 0.66375 \\
\hline$\pi_{7}^{(0)}$ & 0.00000 & 0.00029 & 0.00331 & 0.00011 & 0.00001 & 0.00021 & 0.00003 & 0.00004 & 0.00002 & 0.00001 \\
\hline$\pi_{20}^{(0)}$ & 0.00000 & 0.35051 & 0.39377 & 0.42155 & 0.44240 & 0.46617 & 0.48847 & 0.50541 & 0.53412 & 0.54837 \\
\hline \hline$p^{(0)}$ & 0.00000 & 0.05000 & 0.10000 & 0.15000 & 0.20000 & 0.25000 & 0.30000 & 0.35000 & 0.40000 & 0.45000 \\
\hline
\end{tabular}

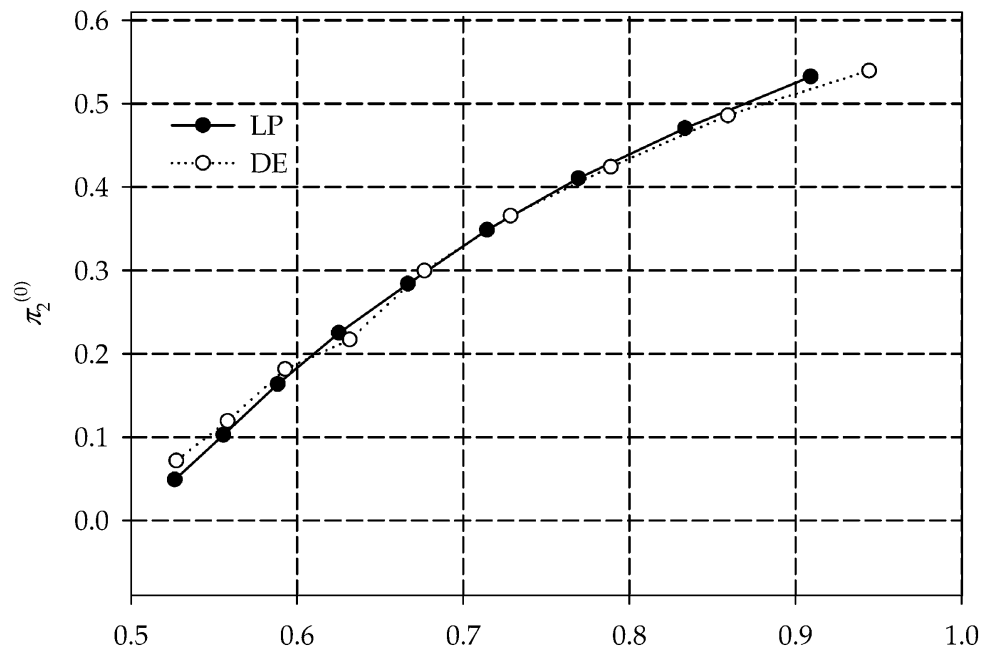

Fig. 15. $\pi_{2}^{(0)}$ 's designed with linear programming and DE for a degree distribution pair, $\lambda(x)=0.23403 x+0.21242 x^{2}+0.14690 x^{5}+0.10284 x^{6}+$ $0.30381 x^{19}$, and $\rho(x)=0.71875 x^{7}+0.28125 x^{8}$.

tured symbols in each group $\psi_{j, k}=\pi_{j}^{(0)}\left(R_{k}\right)\left|G_{j}\right|$ must be nondecreasing with the growing rate, where $R_{k}$ is a rate, which is satisfied except for $\psi_{4, k}$. To make the LDPC codes rate compatible, we determine $\psi_{j, k}$ as follows:

$$
\begin{array}{ll}
\psi_{j, 0} & =0 \\
\psi_{j, k} & = \begin{cases}\pi_{j}^{(0)}\left(R_{k}\right)\left|G_{j}\right|, & \text { when } \pi_{j}^{(0)}\left(R_{k}\right)>\pi_{j}^{(0)}\left(R_{k-1}\right) \\
\psi_{j, k-1}, & \text { otherwise }\end{cases}
\end{array}
$$

where $R_{k-1}<R_{k}$, and $R_{0}$ is the base code rate.

We measure $E_{b} / N_{0}$ gaps between the simulation results as we did in Fig. 11 for the asymptotic $E_{b} / N_{0}$ thresholds of the LDPC code. The gaps between the simulation results and theoretical predictions are compared in Fig. 12, which shows a consistency between them. We also plot the BER curves of the intentionally and randomly punctured LDPC codes in Figs. 13 and 14, respectively.

In addition, we design another puncturing distribution with a 1/2-rate LDPC code from [11] which has a degree distribution pair

$$
\begin{aligned}
\lambda(x)= & 0.23403 x+0.21242 x^{2}+0.14690 x^{5}+0.10284 x^{6} \\
& +0.30381 x^{19}
\end{aligned}
$$

and

$$
\rho(x)=0.71875 x^{7}+0.28125 x^{8} .
$$

The puncturing distributions are obtained with linear programming and listed in Table III. Again, $\pi_{7}^{(0)}$ does not increase with the growing rate. However, the magnitude of $\pi_{7}^{(0)}$ is so small that it can be neglected over the entire range of rates.

We also design the puncturing distributions with the combination of DE and DDE techniques. The puncturing proportions for the symbols in $G_{2}$ are shown in Fig. 15, where the proportions from LP and DE have a good match. Those of the higher degrees have some discrepancies between results from LP and DE. Although we can have better puncturing distributions with DE, as we have explained, the improvement through DE is not distinctive. We implement the punctured LDPC codes with a block length of 131072 and evaluate their $E_{b} / N_{0}$ thresholds which are compared with asymptotic $E_{b} / N_{0}$ thresholds in Fig. 16 . The comparison shows that the simulation results are consistent with the theoretical predictions. The asymptotic $E_{b} / N_{0}$ thresholds with the puncturing distributions acquired through DE show that we can change the rate up to 0.86 at the expense of $0.1 \mathrm{~dB}$. In this experiment, the maximum rate is 0.95 and requires an additional $E_{b} / N_{0}$ gap of $0.3 \mathrm{~dB}$ over that of the base code.

\section{CONCLUSION}

We investigate good puncturing distributions for rate-compatible punctured LDPC codes. The theoretical performance of punctured LDPC codes is analyzed with GA. The analytic results give us an insight into the convergence of punctured LDPC codes and enable us to predict the asymptotic thresholds of punctured LDPC codes. From the analysis, we can see that the residual puncturing fraction during iterations depends only on the structure of a punctured LDPC code specified by a 3-tuple degree distribution $\left(\lambda(x), \rho(x), \pi^{(0)}(x)\right)$ instead of the SNR, which simplifies the recursive equation for the updated mean $m_{u}^{(k)}$ to a steady-state equation. The steady-state equation is a useful tool to visualize how a puncturing distribution works with an LDPC code and how we can design a puncturing distribution to get a smaller $E_{b} / N_{0}$ threshold for a puncturing fraction $p^{(0)}$ or a larger puncturing fraction for a given $E_{b} / N_{0}$. 


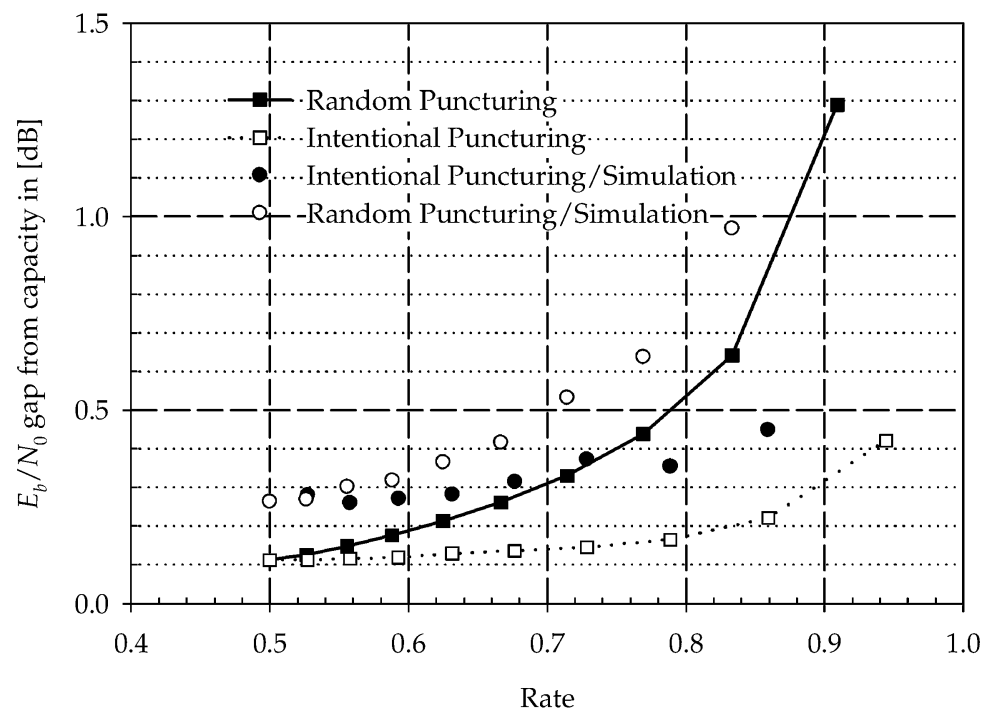

Fig. 16. Gaps between asymptotic $E_{b} / N_{0}$ 's and the capacity of BPSK signal with respect to the rates for a degree distribution pair, $\lambda(x)=0.23403 x+$ $0.21242 x^{2}+0.14690 x^{5}+0.10284 x^{6}+0.30381 x^{19}$ and $\rho(x)=0.71875 x^{7}+0.28125 x^{8}$.

Based on the analysis, we propose a design rule for good puncturing distributions, and the design rule is stated as a simple linear programming problem. Good puncturing distributions can also be designed with a combination of DDE and DE, where DDE models the message-passing decoders more accurately. However, DDE relies only on the numerical computations and cannot give us an analytic insight.

To verify the design rule based on GA, we compare $E_{b} / N_{0}$ thresholds of punctured LDPC codes designed with GA with the ones designed with DDE. The comparison shows that there is less than 0.1-dB discrepancy between the results of two different design rules. The computational advantage of the proposed design rule can offset the difference. If more improved results are needed at the expense of greater complexity, we can use the results from the proposed design rule as initial values of the DE algorithm to speed up the design process.

Furthermore, we design puncturing distributions for LDPC codes from [7] and [11] which have the maximum variable degrees of 10 and 20 , respectively, and evaluate their performance. The performance of a punctured LDPC code is measured by the gap between $E_{b} / N_{0}$ threshold and the capacity of BPSK with respect to the rate.

In the experiment with the first LDPC code, the gap of the base code is $0.2 \mathrm{~dB}$ and an additional $0.1-\mathrm{dB}$ gap permits us to vary the rate up to 0.72 . The changes of rate up to 0.84 and 0.92 are obtained at the expense of 0.2- and 0.4-dB additional gaps, respectively. In the experiment with the second LDPC code, the gap of the base code is $0.1 \mathrm{~dB}$, and we can change the rate up to 0.86 at the expense of $0.1 \mathrm{~dB}$. The maximum rate is 0.95 that requires $0.3 \mathrm{~dB}$ additional $E_{b} / N_{0}$ to the gap of the base code. The LDPC codes are implemented with a block length of 131072 , and their performance is evaluated. We observe that the theoretical results are good lower bounds on the implemented LDPC codes.

Through the work done in this correspondence, we show the existence of good puncturing distributions for LDPC codes and propose a simple but effective design rule. This work is focusing mainly on the theoretical aspects of the problem but can be further studied for designing practical rate-compatible punctured LDPC codes.

\section{ACKNOWLEDGMENT}

The authors would like to thank Demijan Klinc and Victor Stolpman for their comments on earlier drafts of this correspondence. They would also like to thank the reviewers for their many helpful suggestions.

\section{REFERENCES}

[1] D. M. Mandelbaum, "An adaptive feed-back coding using incremental redundancy," IEEE Trans. Inform. Theory, vol. IT-20, pp. 388-389, May 1974.

[2] J. B. Cain Jr, G. C. Clark, and J. M. Geist, "Punctured convolutional codes of rate $(\boldsymbol{n}-\mathbf{1}) / \boldsymbol{n}$ and simplified maximum likelihood decoding," IEEE Trans. Inform. Theory, vol. IT-25, pp. 97-100, Jan. 1979.

[3] J. Hagenauer, "Rate-compatible punctured convolutional codes (RCPC codes) and their applications," IEEE Trans. Commun., vol. 36, pp. 389-400, Apr. 1988.

[4] L. R. Lugand, D. J. Costello Jr, and R. H. Deng, "Parity retransmission hybrid ARQ using rate $1 / 2$ convolutional codes on a nonstationary channel," IEEE Trans. Commun., vol. 37, pp. 755-765, July 1989.

[5] M. Tanner, "A recursive approach to low complexity codes," IEEE Trans. Inform. Theory, vol. IT-27, pp. 533-547, Sept. 1981.

[6] M. Luby, M. Mitzenmacher, A. Shokrollahi, D. Spielman, and V. Stemann, "Practical loss-resilient codes," in Proc. 29th Annu. ACM Symp. Theory of Computing, 1997, pp. 150-159.

[7] T. J. Richardson, A. Shokrollahi, and R. L. Urbanke, "Design of capacity-approaching irregular low-density parity-check codes," IEEE Trans. Inform. Theory, vol. 47, pp. 619-637, Feb. 2001.

[8] T. J. Richardson and R. L. Urbanke, "The capacity of low-density parity-check codes under message-passing decoding," IEEE Trans. Inform. Theory, vol. 47, pp. 599-618, Feb. 2001.

[9] J. Ha and S. W. McLaughlin, "Analysis and design of LDPCCs over Gaussian channels with erasures," in Proc. IEEE Int. Symp. Information Theory, Lausanne, Switzerland, June/July 2002, p. 30.

[10] S.-Y. Chung, T. J. Richardson, and R. L. Urbanke, "Analysis of sumproduct decoding of low-density parity-check codes using a Gaussian approximation," IEEE Trans. Inform. Theory, vol. 47, pp. 657-670, Feb. 2001.

[11] S.-Y. Chung, "On the construction of some capacity-approaching coding schemes," Ph.D. dissertation, MIT, Cambridge, MA, 2000.

[12] M. Luby, M. Mitzenmacher, A. Shokrollahi, and D. Spielman, "Analysis of low density codes and improved designs using irregular graphs," in Proc. 30th Annu. ACM Symp. Theory of Computing, 1998, pp. 249-258.

[13] S.-Y. Chung, G. D. Forney Jr, T. J. Richardson, and R. L. Urbanke, "On the design of low-density parity-check codes within $0.0045 \mathrm{~dB}$ of the Shannon limit," Electron. Lett., vol. 5, no. 2, pp. 58-60, Feb. 2001.

[14] R. G. Bartle, The Elements of Real Analysis, 2nd ed. New York: Wiley, 1976.

[15] K. Price and R. Storn, "Differential evolution-a simple and efficient heuristic for global optimization over continuous spaces," J. Global Optimiz., vol. 11, pp. 341-359, 1977. 